MSC 34B27

\title{
О СУЩЕСТВОВАНИИ РЕШЕНИЯ ПЕРИОДИЧЕСКОЙ КРАЕВОЙ ЗАДАЧИ ДЛЯ ПОЛУЛИНЕЙНЫХ ДИФФЕРЕНЦИАЛЬНЫХ ВКЛЮЧЕНИЙ ДРОБНОГО ПОРЯДКА ИЗ ИНТЕРВАЛА $(3,4)$ В БАНАХОВЫХ ПРОСТРАНСТВАХ
}

\author{
Г. Г. Петросян, М. С. Сорока \\ (Статья представлена членом редакционной коллегии И. П. Половинкиным) \\ ФГБОУ ВО Воронежский государственный педагогический университет, \\ Воронеж, 394043, Россия
}

E-mail: garikpetrosyan@yandex.ru, marya.afanasowa@yandex.ru

\begin{abstract}
Аннотация. В работе исследуется периодическая краевая задача для класса полулинейных дифференциальных включений дробного порядка из интервала $(3,4)$ в банаховом пространстве, для которых многозначная нелинейность удовлетворяет условию регулярности, выраженному в терминах мер некомпактности. Для доказательства существования решения задачи конструируется соответствующая функция Грина. Затем вводится в рассмотрение многозначный разрешающий оператор в пространстве непрерывных функций. После чего поставленная задача сводится к задаче существования неподвижных точек разрешающего мультиоператора. Для доказательства существования неподвижных точек используется обобщенная теорема типа Б. Н. Садовского.
\end{abstract}

Ключевые слова: дифференциальное включение, дробная производная, функция Грина, уплотняющий мультиоператор, мера некомпактности, неподвижная точка.

Благодарности: Работа выполнена при финансовой поддержке Министерства просвещения России в рамках выполнения государственного задания в сфере науки (номер темы FZGF-2020-0009) и гранта Президента РФ для государственной поддержки молодых российских ученых - кандидатов наук, проект МК-338.2021.1.1.

Для цитирования: Петросян Г. Г., Сорока М. С. 2021. О существовании решения периодической краевой задачи для полулинейных дифференциальных включений дробного порядка из интервала $(3,4)$ в банаховых пространствах. Прикладная математика \& Физика. 53(4): 266-283. DOI 10.52575/2687-0959-2021-53-4-266-283.

\section{ON THE EXISTENCE OF A SOLUTION OF A PERIODIC BOUNDARY VALUE PROBLEM FOR SEMILINEAR DIFFERENTIAL INCLUSIONS OF FRACTIONAL ORDER FROM THE INTERVAL $(3,4)$ IN BANACH SPACES}

\section{Garik Petrosyan, Maria Soroka}

(Article submitted by a member of the editorial board I. P. Polovinkin)

Voronezh State Pedagogical University, Voronezh, 394043, Russia

E-mail: garikpetrosyan@yandex.ru, marya.afanasowa@yandex.ru

Received November, 21, 2021

\begin{abstract}
In this paper we study a periodic boundary value problem for a class of semilinear differential inclusions of fractional order from the interval $(3,4)$ in a Banach space for which the multivalued nonlinearity satisfies the regularity condition expressed in terms of measures of noncompactness. We prove the existence of a solution to the problem, we first construct the corresponding Green's function. Then we introduce into consideration a multivalued resolving operator in the space of continuous functions and reduce the problem posed to the problem of the existence of fixed points of a resolving multioperator. We prove the existence of fixed points, by using a generalized B.N. Sadovskii type theorem.
\end{abstract}

Key words: differential inclusion, fractional derivative, Green's function, condensing multioperator, measure of noncompactness, fixed point.

Acknowledgements: The work was supported by the State contract of the Russian Ministry of Education as part of the state task (contract FZGF-2020-0009) and the grant from the President of the Russian Federation for young scientists - candidates of science, project number MK-338.2021.1.1.

For citation: Petrosyan Garik, Soroka Maria. 2021. On the existence of a solution of a periodic boundary value problem for semilinear differential inclusions of fractional order from the interval $(3,4)$ in Banach spaces. Applied Mathematics \& Physics. 53(4): 266-283. (in Russian) DOI 10.52575/2687-0959-2021-53-4-266-283.

1. Введение. Дробное исчисление и теория дифференциальных уравнений и включений дробного порядка приобрели значительную популярность и важность в течение последних трех десятилетий, в 
основном благодаря продемонстрированным приложениям в многочисленных, казалось бы, разнообразных и широко распространенных областях науки и инженерии (см. монографии [19], [22], статьи [1], [2], [3], [14], [17], [18]). Данный раздел математики действительно предоставляет множество полезных инструментов для различных задач, связанных со специальными функциями математической физики, а также их расширениями и обобщениями для одной или нескольких переменных (см. работы [20], [23], [24]). Помимо этого, область применения дробного анализа в современной науке включает исследования в теории потоков жидкости, реологии, динамических процессов в самоподобных и пористых структурах, диффузионного переноса, электросетей, теории управления динамическими системами, теории вязкоупругости, электрохимии оптики и многом другом (см. монографии [11], [25], статью [4]). Одной из важных задач качественного исследования, напрямую связанной с прикладной проблематикой, является задача существования периодических траекторий систем (см., например, [5], [6], [7], [12], [13]). В настоящей работе данная задача решается для класса полулинейных дифференциальных включений дробного порядка $q \in(3,4)$ в банаховом пространстве, для которых многозначная нелинейность удовлетворяет условию регулярности, выраженному в терминах мер некомпактности.

В сепарабельном банаховом пространстве $E$ мы исследуем периодическую краевую задачу для полулинейного дифференциального включения следующего вида:

$$
\begin{gathered}
{ }^{C} D^{q} x(t) \in \eta x(t)+F(t, x(t)), \quad t \in[0, T], \\
x(0)=x(T), \quad x^{\prime}(0)=x^{\prime}(T), \quad x^{\prime \prime}(0)=x^{\prime \prime}(T), \quad x^{\prime \prime \prime}(0)=x^{\prime \prime \prime}(T) .
\end{gathered}
$$

Здесь ${ }^{C} D^{q}$ - дробная производная Герасимова - Капуто, число $\eta>0$, и $F:[0, T] \times E \multimap E-$ многозначное отображение. Отметим, что для полулинейного случая с дробной производной порядка $q \in(3,4)$ такого рода задачи до настоящего времени не были исследованы.

Статья организована следующим образом. Во втором разделе мы приводим необходимые понятия и факты из дробного анализа и теории уплотняющих отображений. В третьем пункте мы конструируем функцию Грина для указанной выше задачи, затем мы вводим и исследуем разрешающий интегральный оператор в пространстве непрерывных функций, неподвижные точки которого совпадают с решениями задачи. На этой основе мы доказываем основной результат существования периодического решения (теорема 4.1).

2. Предварительные сведения. 2.1. Дробный анализ. Вначале приведем известные понятия и утверждения из дробного анализа (см. $[19,22])$.

Определение 2.1. Дробным интегралом порядка $q>0$ функции $g:[0, T] \rightarrow \mathbb{R}$ называется функция $I^{q} g$ следующего вида:

$$
\begin{gathered}
I^{q} g(t)=\frac{1}{\Gamma(q)} \int_{0}^{t}(t-s)^{q-1} g(s) d s, \quad \text { где } \quad \Gamma-\text { гамма-функиия Эйлера } \\
\Gamma(q)=\int_{0}^{\infty} x^{q-1} e^{-x} d x .
\end{gathered}
$$

Для гамма-функции Эйлера выполняется следующее условие, необходимое нам в дальнейшем (см., например, [22]):

$$
\frac{1}{\Gamma(q)}=0, \text { для } q=0,-1,-2, \ldots
$$

Определение 2.3. Дробной производной Герасимова - Капуто порядка $q \geq 0$ функиии $g \in C^{n}([0, T])$ называется функция ${ }^{C} D^{q} \mathrm{~g}$ следующего вида:

$$
{ }^{C} D^{q} g(t)=\frac{1}{\Gamma(n-q)} \int_{0}^{t}(t-s)^{n-q-1} g^{(n)}(s) d s, \quad n=[q]+1 .
$$

Определение 2.4. Функция вида

$$
E_{q, \beta}(z)=\sum_{n=0}^{\infty} \frac{z^{n}}{\Gamma(q n+\beta)}, \quad q>0, \beta \in \mathbb{C}, z \in \mathbb{C},
$$

называется функцией Миттаг - Леффлера.

Зачастую функцию $E_{q, 1}$ обозначают через $E_{q}$. Функция Миттаг - Леффлера имеет огромное значение в теории дифференциальных уравнений дробного порядка. Рассмотрим задачу Коши для скалярного дифференциального уравнения дробного порядка

$$
{ }^{C} D^{q} x(t)=\eta x(t)+f(t), \quad t \in[0, T], 2<q<3,
$$




$$
x(0)=c_{1}, x^{\prime}(0)=c_{2}, x^{\prime \prime}(0)=c_{3}, x^{\prime \prime \prime}(0)=c_{4},
$$

где $\eta \in \mathbb{R}, f:[0, T] \rightarrow \mathbb{R}$ - функция, для которой существует дробный интеграл порядка $q$.

Решением последней задачи является непрерывная функция $x:[0, T] \rightarrow \mathbb{R}$, удовлетворяющая условиям (5), для которой дробная производная Герасимова - Капуто ${ }^{C} D^{q} x$ также непрерывна и удовлетворяет уравнению (4). Известно (см. [19]), что единственным решением данной задачи является функция

$$
x(t)=c_{1} E_{q}\left(\eta t^{q}\right)+c_{2} t E_{q, 2}\left(\eta t^{q}\right)+c_{3} t^{2} E_{q, 3}\left(\eta t^{q}\right)+c_{4} t^{3} E_{q, 4}\left(\eta t^{q}\right)+\int_{0}^{t}(t-s)^{q-1} E_{q, q}\left(\eta(t-s)^{q}\right) f(s) d s .
$$

Справедливы следующие соотношения и утверждение (см. [10]):

$$
\begin{gathered}
E_{q, \beta}(t)=\frac{1}{\Gamma(\beta)}+t E_{q, \beta+q}(t), \\
\left(\frac{d}{d t}\right)^{n}\left(t^{\beta-1} E_{q, \beta}\left(\eta t^{q}\right)\right)=t^{\beta-n-1} E_{q, \beta-n}\left(\eta t^{q}\right), \\
\int_{0}^{z} t^{\beta-1} E_{q, \beta}\left(\eta t^{q}\right) d t=z^{\beta} E_{q, \beta+1}\left(\eta z^{q}\right) .
\end{gathered}
$$

Лемма 2.1. (см. [16]). Для функизи $f \in L^{\infty}([0, T] ; E)$ u $q>1, \beta \in \mathbb{R}$, имеет место равенство

$$
\left(\int_{0}^{t}(t-s)^{q-1} E_{q, \beta}\left(\eta(t-s)^{q}\right) f(s) d s\right)_{t}^{\prime}=\int_{0}^{t}(t-s)^{q-2} E_{q, \beta-1}\left(\eta(t-s)^{q}\right) f(s) d s .
$$

2.2. Многозначные отображения. Пусть $\mathcal{E}$ - банахово пространство. Введем следующие обозначения:

- $P(\mathcal{E})=\{A \subseteq \mathcal{E}: A \neq \varnothing\}$;

- $P b(\mathcal{E})=\{A \in P(\mathcal{E}): A$ ограничено $\}$;

- $\operatorname{Pv}(\mathcal{E})=\{A \in P(\mathcal{E}): A$ выпукло $\}$;

- $K(\mathcal{E})=\{A \in P b(\mathcal{E}): A$ компактно $\}$

- $K v(\mathcal{E})=\operatorname{Pv}(\mathcal{E}) \cap K(\mathcal{E})$.

Определение 2.5. (См., например, $[15,21])$. Пусть ( $\mathcal{A}, \geq)$ частично-упорядоченное множество. Функция $\beta: P b(\mathcal{E}) \rightarrow \mathcal{A}$ называется мерой некомпактности $($ МНК) в $\mathcal{E}$, если для каждого $\Omega \in P b(\mathcal{E})$ выполняется:

$$
\beta(\overline{\mathrm{co}} \Omega)=\beta(\Omega),
$$

где $\overline{\mathrm{co}} \Omega$ обозначает замыкание выпуклой оболочки $\Omega$.

Мера некомпактности $\beta$ называется:

1) монотонной, если для любых $\Omega_{0}, \Omega_{1} \in P b(\mathcal{E})$, включение $\Omega_{0} \subseteq \Omega_{1}$ влечет неравенство $\beta\left(\Omega_{0}\right) \leq$ $\beta\left(\Omega_{1}\right)$;

2) несингулярной, если для любого $a \in \mathcal{E}$ и любого $\Omega \in P b(\mathcal{E})$ выполняется равенство $\beta(\{a\} \cup \Omega)=\beta(\Omega)$.

Если $\mathcal{A}$ конус в банаховом пространстве, то мера некомпактности $\beta$ называется:

3) правильной, если равенство $\beta(\Omega)=0$ эквивалентно относительной компактности множества $\Omega \in$ $\operatorname{Pb}(\mathcal{E})$;

4) вещественной, если $\mathcal{A}$ подмножество действительных чисел $\mathbb{R}$ с естественным порядком;

5) алгебраччески полуаддитивной, если $\beta\left(\Omega_{0}+\Omega_{1}\right) \leq \beta\left(\Omega_{0}\right)+\beta\left(\Omega_{1}\right)$, для всех $\Omega_{0}, \Omega_{1} \in P b(\mathcal{E})$.

Примером вещественной МНК, удовлетворяющей всем вышеперечисленным свойствам, является МНК Хаусдорфа $\chi(\Omega): \chi(\Omega)=\inf \{\varepsilon>0$, для которых $\Omega$ имеет конечную $\varepsilon$-сеть в $\mathcal{E}\}$.

Отметим, что МНК Хаусдорфа удовлетворяет также свойству полуоднородности $\chi(\eta \Omega)=|\eta| \chi(\Omega)$, для всех $\eta \in \mathbb{R}$ и $\Omega \in P b(\mathcal{E})$. Более того, если $\mathcal{L}: \mathcal{E} \rightarrow \mathcal{E}$ - линейный ограниченный оператор, то $\chi(\mathcal{L}(\Omega))=\|\mathcal{L}\| \chi(\Omega)$ для любого $\Omega \in P b(\mathcal{E})$. Норма множества $M \in P b(\mathcal{E})$ определяется по формуле $\|M\|=\sup _{x \in M}\|x\|_{\mathcal{E}}$.

Следующие понятия и утверждения можно найти в монографиях $[15,21]$. 
Определение 2.6. Пусть $X$ - метрическое пространство. Многозначное отображение (мультиотображение) $\mathcal{F}: X \rightarrow P(\mathcal{E})$ называется:

(i) полунепрерывным сверху (п.н.с.), если $\mathcal{F}^{-1}(V)=\{x \in X: \mathcal{F}(x) \subset V\}$ - открытое подмножество $X$ для любого открытого множества $V \subset \mathcal{E}$,

(ii) замкнутым, если график $\Gamma_{\mathcal{F}}=\{(x, y): y \in \mathcal{F}(x)\}-$ замкнутое подмножество $X \times \mathcal{E}$,

(iii) компактным, если $\mathcal{F}(X)$ - относительно компактно в $\mathcal{E}$,

(iv) квазикомпактным, если сужение на любое компактное подмножество $A \subset X$ компактно.

Лемма 2.2. Пусть $X$ и $Y$-метрические пространства и $\mathcal{F}: X \rightarrow K(Y)$ - замкнутое квазикомпактное мультиотображение, тогда $\mathcal{F}-$ п.н.с.

Определение 2.7. Мультиотображение $\mathcal{F}: X \subseteq \mathcal{E} \rightarrow K(\mathcal{E})$ называется уплотняющим относительно МНК $\beta$ ( $\beta$ - уплотняющим), если для любого ограниченного множества $\Omega \subseteq X$, не являющегося относительно компактным, выполнено:

$$
\beta(F(\Omega)) \nsupseteq \beta(\Omega) .
$$

Справедлива следующая теорема о неподвижной точке для уплотняющих мультиотображений (см., например, [15, 21]).

Теорема 2.1. Пусть $\mathcal{M}$ - выпуклое замкнутое подмножество $\mathcal{E}$ и $\mathcal{F}: \mathcal{M} \rightarrow K v(\mathcal{M})$ - $\beta$ - уплотняющее мультиотображение, где $\beta$ - несингулярная мера некомпактности в $\mathcal{E}$. Тогда множество неподвижных точек $\mathcal{F}:$ Fix $\mathcal{F}:=\{x: x \in \mathcal{F}(x)\}-$ непустое множество.

2.3. Измеримые мультифункции. Напомним некоторые понятия (см., например, [15, 21]). Пусть E - банахово пространство.

Определение 2.8. Мультифункция $G:[0, T] \rightarrow K(E)$, для $p \geq 1$, называется:

- $L^{p}$-интегрируемой, если она допускает $L^{p}$-интегрируемое сечение по Бохнеру, т.е. существует функиия $g \in L^{p}([0, T] ; E)$ такая, что $g(t) \in G(t)$ для п. в. $t \in[0, T]$;

- $L^{p}$-интегрально ограниченной, если существует функция $\xi \in L^{p}([0, T])$ такая, что:

$$
\|G(t)\|:=\sup \left\{\|g\|_{E}: g(t) \in G(t)\right\} \leq \xi(t)
$$

для п. в. $t \in[0, T]$.

Множество всех $L^{p}$-интегрируемых сечений мультифункции $G:[0, T] \rightarrow K(E)$ обозначается $\mathcal{S}_{G}^{p}$.

Определение 2.9. Последовательность функиий $\left\{\xi_{n}\right\} \subset L^{p}([0, T] ; E), p \geq 1$, называется $L^{p}$-полукомпактной, если она $L^{p}$-интегрально ограничена, то есть

$$
\left\|\xi_{n}(t)\right\|_{E} \leq v(t) \text { для всех } n=1,2, \ldots, \text { и п.в. } t \in[0, T],
$$

где v $\in L_{+}^{p}([0, T])$ имножество $\left\{\xi_{n}(t)\right\}$ относительно компактно в Е для п.в. $t \in[0, T]$.

3. Основные результаты

Рассмотрим в сепарабельном банаховом пространстве $E$ краевую задачу (4), (5):

$$
\begin{gathered}
{ }^{c} D^{q} x(t)=\eta x(t)+f(t), \quad t \in[0, T], 3<q<4, \\
x(0)=c_{1}, x^{\prime}(0)=c_{2}, x^{\prime \prime}(0)=c_{3}, x^{\prime \prime \prime}(0)=c_{4}, \quad \text { где } \quad f:[0, T] \rightarrow E .
\end{gathered}
$$

Определение 3.1. Интегральным решением краевой задачи (4), (5) называется функция $x \in C([0, T] ; E)$, удовлетворяющая равенству

$$
x(t)=c_{1} E_{q}\left(\eta t^{q}\right)+c_{2} t E_{q, 2}\left(\eta t^{q}\right)+c_{3} t^{2} E_{q, 3}\left(\eta t^{q}\right)+c_{4} t^{3} E_{q, 4}\left(\eta t^{q}\right)+\int_{0}^{t}(t-s)^{q-1} E_{q, q}\left(\eta(t-s)^{q}\right) f(s) d s .
$$

Лемма 3.1. Пycmb $f \in C([0, T] ; E) u$

$$
\begin{gathered}
\alpha=\left(1-E_{q}\left(\eta T^{q}\right)\right)^{4}-2\left(1-E_{q}\left(\eta T^{q}\right)\right) E_{q, 3}\left(\eta T^{q}\right) E_{q, 0}^{2}\left(\eta T^{q}\right)-2\left(1-E_{q}\left(\eta T^{q}\right)\right) E_{q, 2}^{2}\left(\eta T^{q}\right) E_{q,-1}\left(\eta T^{q}\right)- \\
2\left(1-E_{q}\left(\eta T^{q}\right)\right)^{2} E_{q, 3}\left(\eta T^{q}\right) E_{q,-1}\left(\eta T^{q}\right)-3\left(1-E_{q}\left(\eta T^{q}\right)\right)^{2} E_{q, 2}\left(\eta T^{q}\right) E_{q, 0}\left(\eta T^{q}\right)-E_{q, 2}^{3}\left(\eta T^{q}\right) E_{q,-2}\left(\eta T^{q}\right)- \\
2 E_{q, 2}\left(\eta T^{q}\right) E_{q, 3}\left(\eta T^{q}\right) E_{q,-1}\left(\eta T^{q}\right) E_{q, 0}\left(\eta T^{q}\right)-2 E_{q, 2}\left(\eta T^{q}\right) E_{q, 3}\left(\eta T^{q}\right) E_{q,-2}\left(\eta T^{q}\right)\left(1-E_{q}\left(\eta T^{q}\right)\right)+ \\
E_{q, 2}^{2}\left(\eta T^{q}\right) E_{q, 0}^{2}\left(\eta T^{q}\right)+E_{q, 3}^{2}\left(\eta T^{q}\right) E_{q,-1}^{2}\left(\eta T^{q}\right)-E_{q, 3}^{2}\left(\eta T^{q}\right) E_{q, 0}\left(\eta T^{q}\right) E_{q,-2}\left(\eta T^{q}\right)-E_{q, 4}\left(\eta T^{q}\right) E_{q, 0}^{3}\left(\eta T^{q}\right)- \\
E_{q, 4}\left(\eta T^{q}\right) E_{q,-2}\left(\eta T^{q}\right)\left(1-E_{q}\left(\eta T^{q}\right)\right)^{2}-E_{q, 4}\left(\eta T^{q}\right) E_{q, 2}\left(\eta T^{q}\right) E_{q,-1}^{2}\left(\eta T^{q}\right)+ \\
E_{q, 4}\left(\eta T^{q}\right) E_{q, 2}\left(\eta T^{q}\right) E_{q, 0}\left(\eta T^{q}\right) E_{q,-2}\left(\eta T^{q}\right)-E_{q, 4}\left(\eta T^{q}\right) E_{q, 0}\left(\eta T^{q}\right) E_{q,-1}\left(\eta T^{q}\right)\left(1-E_{q}\left(\eta T^{q}\right)\right)- \\
\left(1-E_{q}\left(\eta T^{q}\right)\right) E_{q, 4}\left(\eta T^{q}\right) E_{q,-1}\left(\eta T^{q}\right) E_{q, 0}\left(\eta T^{q}\right) \neq 0 .
\end{gathered}
$$


Тогда краевая задача (4),(2):

$$
\begin{gathered}
{ }^{C} D^{q} x(t)=\eta x(t)+f(t), \quad t \in[0, T], 3<q<4, \\
x(0)=x(T), x^{\prime}(0)=x^{\prime}(T), x^{\prime \prime}(0)=x^{\prime \prime}(T), x^{\prime \prime \prime}(0)=x^{\prime \prime \prime}(T)
\end{gathered}
$$

имеет единственное решение

$$
x(t)=\int_{0}^{T} G(t, s) f(s) d s,
$$

где функция Грина $G(t, s)$ имеет следующий вид

$$
G(t, s)=
$$$$
\left\{\begin{array}{l}
\alpha^{-1}\left(E_{q}\left(\eta t^{q}\right) \alpha_{1}+t E_{q, 2}\left(\eta t^{q}\right) T^{-1} \alpha_{5}+t^{2} E_{q, 3}\left(\eta t^{q}\right) T^{-2} \alpha_{8}+t^{3} E_{q, 4}\left(\eta t^{q}\right) T^{-3} \alpha_{10}\right)(T-s)^{q-1} E_{q, q}\left(\eta(T-s)^{q}\right)+ \\
\alpha^{-1}\left(E_{q}\left(\eta t^{q}\right) T \alpha_{2}+t E_{q, 2}\left(\eta t^{q}\right) \alpha_{6}+t^{2} E_{q, 3}\left(\eta t^{q}\right) T^{-1} \alpha_{9}+t^{3} E_{q, 4}\left(\eta t^{q}\right) T^{-2} \alpha_{8}\right)(T-s)^{q-2} E_{q, q-1}\left(\eta(T-s)^{q}\right)+ \\
\alpha^{-1}\left(E_{q}\left(\eta t^{q}\right) T^{2} \alpha_{3}+t E_{q, 2}\left(\eta t^{q}\right) T \alpha_{7}+t^{2} E_{q, 3}\left(\eta t^{q}\right) \alpha_{6}+t^{3} E_{q, 4}\left(\eta t^{q}\right) T^{-1} \alpha_{5}\right)(T-s)^{q-3} E_{q, q-2}\left(\eta(T-s)^{q}\right)+ \\
\alpha^{-1}\left(E_{q}\left(\eta t^{q}\right) T^{3} \alpha_{4}+t E_{q, 2}\left(\eta t^{q}\right) T^{2} \alpha_{3}+t^{2} E_{q, 3}\left(\eta t^{q}\right) T \alpha_{2}+t^{3} E_{q, 4}\left(\eta t^{q}\right) \alpha_{1}\right)(T-s)^{q-4} E_{q, q-3}\left(\eta(T-s)^{q}\right)+ \\
(t-s)^{q-1} E_{q, q}\left(\eta(t-s)^{q}\right), \quad 0 \leq s \leq t \leq T, \\
\alpha^{-1}\left(E_{q}\left(\eta t^{q}\right) \alpha_{1}+t E_{q, 2}\left(\eta t^{q}\right) T^{-1} \alpha_{5}+t^{2} E_{q, 3}\left(\eta t^{q}\right) T^{-2} \alpha_{8}+t^{3} E_{q, 4}\left(\eta t^{q}\right) T^{-3} \alpha_{10}\right)(T-s)^{q-1} E_{q, q}\left(\eta(T-s)^{q}\right)+ \\
\alpha^{-1}\left(E_{q}\left(\eta t^{q}\right) T \alpha_{2}+t E_{q, 2}\left(\eta t^{q}\right) \alpha_{6}+t^{2} E_{q, 3}\left(\eta t^{q}\right) T^{-1} \alpha_{9}+t^{3} E_{q, 4}\left(\eta t^{q}\right) T^{-2} \alpha_{8}\right)(T-s)^{q-2} E_{q, q-1}\left(\eta(T-s)^{q}\right)+ \\
\alpha^{-1}\left(E_{q}\left(\eta t^{q}\right) T^{2} \alpha_{3}+t E_{q, 2}\left(\eta t^{q}\right) T \alpha_{7}+t^{2} E_{q, 3}\left(\eta t^{q}\right) \alpha_{6}+t^{3} E_{q, 4}\left(\eta t^{q}\right) T^{-1} \alpha_{5}\right)(T-s)^{q-3} E_{q, q-2}\left(\eta(T-s)^{q}\right)+ \\
\alpha^{-1}\left(E_{q}\left(\eta t^{q}\right) T^{3} \alpha_{4}+t E_{q, 2}\left(\eta t^{q}\right) T^{2} \alpha_{3}+t^{2} E_{q, 3}\left(\eta t^{q}\right) T \alpha_{2}+t^{3} E_{q, 4}\left(\eta t^{q}\right) \alpha_{1}\right)(T-s)^{q-4} E_{q, q-3}\left(\eta(T-s)^{q}\right), \\
0 \leq t<s<T,
\end{array}\right.
$$

$$
\begin{gathered}
\alpha_{1}=\left(1-E_{q}\left(\eta T^{q}\right)\right)^{3}-E_{q, 3}\left(\eta T^{q}\right) E_{q, 0}^{2}\left(\eta T^{q}\right)-E_{q, 2}^{2}\left(\eta T^{q}\right) E_{q,-1}\left(\eta T^{q}\right)- \\
E_{q, 3}\left(\eta T^{q}\right) E_{q,-1}\left(\eta T^{q}\right)\left(1-E_{q}\left(\eta T^{q}\right)\right)-2 E_{q, 2}\left(\eta T^{q}\right) E_{q, 0}\left(\eta T^{q}\right)\left(1-E_{q}\left(\eta T^{q}\right)\right),
\end{gathered}
$$

$$
\begin{gathered}
\alpha_{2}=E_{q, 2}\left(\eta T^{q}\right)\left(1-E_{q}\left(\eta T^{q}\right)\right)^{2}-E_{q, 2}^{2}\left(\eta T^{q}\right) E_{q, 0}\left(\eta T^{q}\right)+E_{q, 3}\left(\eta T^{q}\right) E_{q, 0}\left(\eta T^{q}\right)\left(1-E_{q}\left(\eta T^{q}\right)\right)+ \\
E_{q, 4}\left(\eta T^{q}\right) E_{q, 0}^{2}\left(\eta T^{q}\right)+E_{q, 3}\left(\eta T^{q}\right) E_{q, 2}\left(\eta T^{q}\right) E_{q,-1}\left(\eta T^{q}\right)+E_{q, 4}\left(\eta T^{q}\right) E_{q,-1}\left(\eta T^{q}\right)\left(1-E_{q}\left(\eta T^{q}\right)\right) \\
\alpha_{3}=E_{q, 2}\left(\eta T^{q}\right) E_{q, 3}\left(\eta T^{q}\right) E_{q, 0}\left(\eta T^{q}\right)-E_{q, 3}^{2}\left(\eta T^{q}\right) E_{q,-1}\left(\eta T^{q}\right)+E_{q, 3}\left(\eta T^{q}\right)\left(1-E_{q}\left(\eta T^{q}\right)\right)^{2}+ \\
E_{q, 4}\left(\eta T^{q}\right) E_{q, 2}\left(\eta T^{q}\right) E_{q,-1}\left(\eta T^{q}\right)+E_{q, 2}^{2}\left(\eta T^{q}\right)\left(1-E_{q}\left(\eta T^{q}\right)\right)+E_{q, 4}\left(\eta T^{q}\right) E_{q, 0}\left(\eta T^{q}\right)\left(1-E_{q}\left(\eta T^{q}\right)\right) \\
\alpha_{4}=E_{q, 2}^{3}\left(\eta T^{q}\right)-E_{q, 4}\left(\eta T^{q}\right) E_{q, 2}\left(\eta T^{q}\right) E_{q, 0}\left(\eta T^{q}\right)+E_{q, 3}^{2}\left(\eta T^{q}\right) E_{q, 0}\left(\eta T^{q}\right)+E_{q, 4}\left(\eta T^{q}\right)\left(1-E_{q}\left(\eta T^{q}\right)\right)^{2}+ \\
\alpha_{5}=E_{q, 0}\left(\eta T^{q}\right)\left(1-E_{q}\left(\eta T^{q}\right)\right)^{2}+E_{q, 2}^{2}\left(\eta T^{q}\right) E_{q,-2}\left(\eta T^{q}\right)+E_{q, 3}\left(\eta T^{q}\right) E_{q,-1}\left(\eta T^{q}\right) E_{q, 0}\left(\eta T^{q}\right)+ \\
E_{q, 3}\left(\eta T^{q}\right) E_{q,-2}\left(\eta T^{q}\right)\left(1-E_{q}\left(\eta T^{q}\right)\right)-E_{q, 0}^{2}\left(\eta T^{q}\right) E_{q, 2}\left(\eta T^{q}\right)+E_{q, 2}\left(\eta T^{q}\right) E_{q,-1}\left(\eta T^{q}\right)\left(1-E_{q}\left(\eta T^{q}\right)\right) \\
\alpha_{6}=\left(1-E_{q}\left(\eta T^{q}\right)\right)^{3}-E_{q, 2}\left(\eta T^{q}\right) E_{q, 0}\left(\eta T^{q}\right)\left(1-E_{q}\left(\eta T^{q}\right)\right)-E_{q, 3}\left(\eta T^{q}\right) E_{q, 2}\left(\eta T^{q}\right) E_{q,-2}\left(\eta T^{q}\right)- \\
E_{q, 3}\left(\eta T^{q}\right) E_{q, 0}\left(\eta T^{q}\right) E_{q,-2}\left(\eta T^{q}\right)+E_{q, 0}\left(\eta T^{q}\right) E_{q, 2}\left(\eta T^{q}\right) E_{q,-1}\left(\eta T^{q}\right)+E_{q,-1}\left(\eta T^{q}\right)\left(1-E_{q}\left(\eta T^{q}\right)\right)^{2} \\
\alpha_{8}=E_{q, 0}^{2}\left(\eta T^{q}\right)\left(1-E_{q}\left(\eta T^{q}\right)\right)-E_{q, 3}\left(\eta T^{q}\right) E_{q,-1}^{2}\left(\eta T^{q}\right)\left(1-E_{q}\left(\eta T^{q}\right)\right)+E_{q, 4}\left(\eta T^{q}\right) E_{q,-2}\left(\eta T^{q}\right)\left(1-E_{q}\left(\eta T^{q}\right)\right)-E_{q, 4}\left(\eta T^{q}\right) E_{q,-1}\left(\eta T^{q}\right) E_{q, 0}\left(\eta T^{q}\right), \\
\alpha_{7}=E_{q, 2}\left(\eta T^{q}\right)\left(1-E_{q}\left(\eta T^{q}\right)\right)^{2}+2 E_{q, 3}\left(\eta T^{q}\right) E_{q, 0}\left(\eta T^{q}\right)\left(1-E_{q}\left(\eta T^{q}\right)\right)+E_{q, 3}^{2}\left(\eta T^{q}\right) E_{q,-2}\left(\eta T^{q}\right)+ \\
E_{q, 4}\left(\eta T^{q}\right) E_{q, 0}^{2}\left(\eta T^{q}\right)-E_{q, 4}\left(\eta T^{q}\right) E_{q, 2}\left(\eta T^{q}\right) E_{q,-2}\left(\eta T^{q}\right), \\
q
\end{gathered}
$$




$$
\begin{gathered}
\alpha_{9}=2 E_{q, 2}\left(\eta T^{q}\right) E_{q,-1}\left(\eta T^{q}\right)\left(1-E_{q}\left(\eta T^{q}\right)\right)+E_{q, 0}\left(\eta T^{q}\right)\left(1-E_{q}\left(\eta T^{q}\right)\right)^{2}+ \\
E_{q, 2}^{2}\left(\eta T^{q}\right) E_{q,-2}\left(\eta T^{q}\right)+E_{q, 4}\left(\eta T^{q}\right) E_{q,-1}^{2}\left(\eta T^{q}\right)-E_{q, 4}\left(\eta T^{q}\right) E_{q, 0}\left(\eta T^{q}\right) E_{q,-2}\left(\eta T^{q}\right), \\
\alpha_{10}=E_{q, 0}^{3}\left(\eta T^{q}\right)+E_{q,-2}\left(\eta T^{q}\right)\left(1-E_{q}\left(\eta T^{q}\right)\right)^{2}+E_{q, 2}\left(\eta T^{q}\right) E_{q,-1}^{2}\left(\eta T^{q}\right)- \\
E_{q, 2}\left(\eta T^{q}\right) E_{q, 0}\left(\eta T^{q}\right) E_{q,-2}\left(\eta T^{q}\right)+2 E_{q, 0}\left(\eta T^{q}\right) E_{q,-1}\left(\eta T^{q}\right)\left(1-E_{q}\left(\eta T^{q}\right)\right) .
\end{gathered}
$$

Доказательство. Решение краевой задачи (4), (5) в банаховом пространстве $E$ имеет следующий вид

$$
x(t)=c_{1} E_{q}\left(\eta t^{q}\right)+c_{2} t E_{q, 2}\left(\eta t^{q}\right)+c_{3} t^{2} E_{q, 3}\left(\eta t^{q}\right)+c_{4} t^{3} E_{q, 4}\left(\eta t^{q}\right)+\int_{0}^{t}(t-s)^{q-1} E_{q, q}\left(\eta(t-s)^{q}\right) f(s) d s .
$$

Применяя формулу (7) и лемму 2.1, мы можем найти

$$
\begin{gathered}
x^{\prime}(t)=c_{1} t^{-1} E_{q, 0}\left(\eta t^{q}\right)+c_{2} E_{q}\left(\eta t^{q}\right)+c_{3} t E_{q, 2}\left(\eta t^{q}\right)+c_{4} t^{2} E_{q, 3}\left(\eta t^{q}\right)+\int_{0}^{t}(t-s)^{q-2} E_{q, q-1}\left(\eta(t-s)^{q}\right) f(s) d s \\
x^{\prime \prime}(t)=c_{1} t^{-2} E_{q,-1}\left(\eta t^{q}\right)+c_{2} t^{-1} E_{q, 0}\left(\eta t^{q}\right)+c_{3} E_{q}\left(\eta t^{q}\right)+c_{4} t E_{q, 2}\left(\eta t^{q}\right)+\int_{0}^{t}(t-s)^{q-3} E_{q, q-2}\left(\eta(t-s)^{q}\right) f(s) d s \\
x^{\prime \prime \prime}(t)=c_{1} t^{-3} E_{q,-2}\left(\eta t^{q}\right)+c_{2} t^{-2} E_{q,-1}\left(\eta t^{q}\right)+c_{3} t^{-1} E_{q, 0}\left(\eta t^{q}\right)+c_{4} E_{q}\left(\eta t^{q}\right)+\int_{0}^{t}(t-s)^{q-4} E_{q, q-3}\left(\eta(t-s)^{q}\right) f(s) d s
\end{gathered}
$$

Заметим, что благодаря свойству $\frac{1}{\Gamma(0)}=\frac{1}{\Gamma(-1)}=\frac{1}{\Gamma(-2)}=0$, для функций $E_{q, 0}\left(\eta t^{q}\right), E_{q,-1}\left(\eta t^{q}\right)$ и $E_{q,-2}\left(\eta t^{q}\right)$ мы имеем

следовательно,

$$
\begin{gathered}
E_{q, 0}\left(\eta t^{q}\right)=\sum_{n=0}^{\infty} \frac{\left(\eta t^{q}\right)^{n}}{\Gamma(q n)}=\frac{1}{\Gamma(0)}+\sum_{n=1}^{\infty} \frac{\left(\eta t^{q}\right)^{n}}{\Gamma(q n)}=\sum_{n=1}^{\infty} \frac{\left(\eta t^{q}\right)^{n}}{\Gamma(q n)}, \\
E_{q,-1}\left(\eta t^{q}\right)=\sum_{n=0}^{\infty} \frac{\left(\eta t^{q}\right)^{n}}{\Gamma(q n-1)}=\frac{1}{\Gamma(-1)}+\sum_{n=1}^{\infty} \frac{\left(\eta t^{q}\right)^{n}}{\Gamma(q n-1)}=\sum_{n=1}^{\infty} \frac{\left(\eta t^{q}\right)^{n}}{\Gamma(q n-1)} \\
E_{q,-2}\left(\eta t^{q}\right)=\sum_{n=0}^{\infty} \frac{\left(\eta t^{q}\right)^{n}}{\Gamma(q n-2)}=\frac{1}{\Gamma(-2)}+\sum_{n=1}^{\infty} \frac{\left(\eta t^{q}\right)^{n}}{\Gamma(q n-2)}=\sum_{n=1}^{\infty} \frac{\left(\eta t^{q}\right)^{n}}{\Gamma(q n-2)}
\end{gathered}
$$

$$
\begin{aligned}
t^{-1} E_{q, 0}\left(\eta t^{q}\right) & =\sum_{n=1}^{\infty} \frac{\eta^{n} t^{q n-1}}{\Gamma(q n)}, \\
t^{-2} E_{q,-1}\left(\eta t^{q}\right) & =\sum_{n=1}^{\infty} \frac{\eta^{n} t^{q n-2}}{\Gamma(q n-1)}, \\
t^{-3} E_{q,-2}\left(\eta t^{q}\right) & =\sum_{n=1}^{\infty} \frac{\eta^{n} t^{q n-3}}{\Gamma(q n-2)} .
\end{aligned}
$$

В силу последних формул, имеем $x(0)=c_{1}, \quad x^{\prime}(0)=c_{2}, \quad x^{\prime \prime}(0)=c_{3} \quad x^{\prime \prime \prime}(0)=c_{4}$.

Теперь, используя условия (2), мы получаем систему

$$
\left\{\begin{array}{c}
c_{1}=c_{1} E_{q}\left(\eta T^{q}\right)+c_{2} T E_{q, 2}\left(\eta T^{q}\right)+c_{3} T^{2} E_{q, 3}\left(\eta T^{q}\right)+c_{4} T^{3} E_{q, 4}\left(\eta T^{q}\right)+ \\
\int_{0}^{T}(T-s)^{q-1} E_{q, q}\left(\eta(T-s)^{q}\right) f(s) d s \\
c_{2}=c_{1} T^{-1} E_{q, 0}\left(\eta T^{q}\right)+c_{2} E_{q}\left(\eta T^{q}\right)+c_{3} T E_{q, 2}\left(\eta T^{q}\right)+c_{4} T^{2} E_{q, 3}\left(\eta T^{q}\right)+ \\
\int_{0}^{T}(T-s)^{q-2} E_{q, q-1}\left(\eta(T-s)^{q}\right) f(s) d s \\
c_{3}=c_{1} T^{-2} E_{q,-1}\left(\eta T^{q}\right)+c_{2} T^{-1} E_{q, 0}\left(\eta T^{q}\right)+c_{3} E_{q}\left(\eta T^{q}\right)+c_{4} T E_{q, 2}\left(\eta T^{q}\right)+ \\
\int_{0}^{T}(T-s)^{q-3} E_{q, q-2}\left(\eta(T-s)^{q}\right) f(s) d s \\
c_{4}=c_{1} T^{-3} E_{q,-2}\left(\eta T^{q}\right)+c_{2} T^{-2} E_{q,-1}\left(\eta T^{q}\right)+c_{3} T^{-1} E_{q, 0}\left(\eta T^{q}\right)+c_{4} E_{q}\left(\eta T^{q}\right)+ \\
\int_{0}^{T}(T-s)^{q-4} E_{q, q-3}\left(\eta(T-s)^{q}\right) f(s) d s
\end{array}\right.
$$

Выпишем определители для решения последней системы, на основе правила Крамера

$$
\begin{aligned}
\Delta= & \left(1-E_{q}\left(\eta T^{q}\right)\right)^{4}-2\left(1-E_{q}\left(\eta T^{q}\right)\right) E_{q, 3}\left(\eta T^{q}\right) E_{q, 0}^{2}\left(\eta T^{q}\right)-2\left(1-E_{q}\left(\eta T^{q}\right)\right) E_{q, 2}^{2}\left(\eta T^{q}\right) E_{q,-1}\left(\eta T^{q}\right)- \\
2(1- & \left.E_{q}\left(\eta T^{q}\right)\right)^{2} E_{q, 3}\left(\eta T^{q}\right) E_{q,-1}\left(\eta T^{q}\right)-3\left(1-E_{q}\left(\eta T^{q}\right)\right)^{2} E_{q, 2}\left(\eta T^{q}\right) E_{q, 0}\left(\eta T^{q}\right)-E_{q, 2}^{3}\left(\eta T^{q}\right) E_{q,-2}\left(\eta T^{q}\right)- \\
& 2 E_{q, 2}\left(\eta T^{q}\right) E_{q, 3}\left(\eta T^{q}\right) E_{q,-1}\left(\eta T^{q}\right) E_{q, 0}\left(\eta T^{q}\right)-2 E_{q, 2}\left(\eta T^{q}\right) E_{q, 3}\left(\eta T^{q}\right) E_{q,-2}\left(\eta T^{q}\right)\left(1-E_{q}\left(\eta T^{q}\right)\right)+
\end{aligned}
$$




$$
\begin{aligned}
& E_{q, 2}^{2}\left(\eta T^{q}\right) E_{q, 0}^{2}\left(\eta T^{q}\right)+E_{q, 3}^{2}\left(\eta T^{q}\right) E_{q,-1}^{2}\left(\eta T^{q}\right)-E_{q, 3}^{2}\left(\eta T^{q}\right) E_{q, 0}\left(\eta T^{q}\right) E_{q,-2}\left(\eta T^{q}\right)-E_{q, 4}\left(\eta T^{q}\right) E_{q, 0}^{3}\left(\eta T^{q}\right)- \\
& E_{q, 4}\left(\eta T^{q}\right) E_{q,-2}\left(\eta T^{q}\right)\left(1-E_{q}\left(\eta T^{q}\right)\right)^{2}-E_{q, 4}\left(\eta T^{q}\right) E_{q, 2}\left(\eta T^{q}\right) E_{q,-1}^{2}\left(\eta T^{q}\right)+ \\
& E_{q, 4}\left(\eta T^{q}\right) E_{q, 2}\left(\eta T^{q}\right) E_{q, 0}\left(\eta T^{q}\right) E_{q,-2}\left(\eta T^{q}\right)-E_{q, 4}\left(\eta T^{q}\right) E_{q, 0}\left(\eta T^{q}\right) E_{q,-1}\left(\eta T^{q}\right)\left(1-E_{q}\left(\eta T^{q}\right)\right)- \\
& \left(1-E_{q}\left(\eta T^{q}\right)\right) E_{q, 4}\left(\eta T^{q}\right) E_{q,-1}\left(\eta T^{q}\right) E_{q, 0}\left(\eta T^{q}\right)=\alpha, \\
& \Delta_{1}=\left(\left(1-E_{q}\left(\eta T^{q}\right)\right)^{3}-E_{q, 3}\left(\eta T^{q}\right) E_{q, 0}^{2}\left(\eta T^{q}\right)\right) \int_{0}^{T}(T-s)^{q-1} E_{q, q}\left(\eta(T-s)^{q}\right) f(s) d s- \\
& \left(E_{q, 2}^{2}\left(\eta T^{q}\right) E_{q,-1}\left(\eta T^{q}\right)+E_{q, 3}\left(\eta T^{q}\right) E_{q,-1}\left(\eta T^{q}\right)\left(1-E_{q}\left(\eta T^{q}\right)\right)\right) \int_{0}^{T}(T-s)^{q-1} E_{q, q}\left(\eta(T-s)^{q}\right) f(s) d s- \\
& 2 E_{q, 2}\left(\eta T^{q}\right) E_{q, 0}\left(\eta T^{q}\right)\left(1-E_{q}\left(\eta T^{q}\right)\right) \int_{0}^{T}(T-s)^{q-1} E_{q, q}\left(\eta(T-s)^{q}\right) f(s) d s+ \\
& T\left(E_{q, 2}\left(\eta T^{q}\right)\left(1-E_{q}\left(\eta T^{q}\right)\right)^{2}-E_{q, 2}^{2}\left(\eta T^{q}\right) E_{q, 0}\left(\eta T^{q}\right)\right) \int_{0}^{T}(T-s)^{q-2} E_{q, q-1}\left(\eta(T-s)^{q}\right) f(s) d s+ \\
& T\left(E_{q, 3}\left(\eta T^{q}\right) E_{q, 0}\left(\eta T^{q}\right)\left(1-E_{q}\left(\eta T^{q}\right)\right)+E_{q, 4}\left(\eta T^{q}\right) E_{q, 0}^{2}\left(\eta T^{q}\right)\right) \int_{0}^{T}(T-s)^{q-2} E_{q, q-1}\left(\eta(T-s)^{q}\right) f(s) d s+ \\
& T\left(E_{q, 3}\left(\eta T^{q}\right) E_{q, 2}\left(\eta T^{q}\right) E_{q,-1}\left(\eta T^{q}\right)+E_{q, 4}\left(\eta T^{q}\right) E_{q,-1}\left(\eta T^{q}\right)\left(1-E_{q}\left(\eta T^{q}\right)\right)\right) \int_{0}^{T}(T-s)^{q-2} E_{q, q-1}\left(\eta(T-s)^{q}\right) f(s) d s+ \\
& T^{2}\left(E_{q, 2}\left(\eta T^{q}\right) E_{q, 3}\left(\eta T^{q}\right) E_{q, 0}\left(\eta T^{q}\right)-E_{q, 3}^{2}\left(\eta T^{q}\right) E_{q,-1}\left(\eta T^{q}\right)\right) \int_{0}^{T}(T-s)^{q-3} E_{q, q-2}\left(\eta(T-s)^{q}\right) f(s) d s+ \\
& T^{2}\left(E_{q, 3}\left(\eta T^{q}\right)\left(1-E_{q}\left(\eta T^{q}\right)\right)^{2}+E_{q, 4}\left(\eta T^{q}\right) E_{q, 2}\left(\eta T^{q}\right) E_{q,-1}\left(\eta T^{q}\right)\right) \int_{0}^{T}(T-s)^{q-3} E_{q, q-2}\left(\eta(T-s)^{q}\right) f(s) d s+ \\
& T^{2}\left(E_{q, 2}^{2}\left(\eta T^{q}\right)\left(1-E_{q}\left(\eta T^{q}\right)\right)+E_{q, 4}\left(\eta T^{q}\right) E_{q, 0}\left(\eta T^{q}\right)\left(1-E_{q}\left(\eta T^{q}\right)\right)\right) \int_{0}^{T}(T-s)^{q-3} E_{q, q-2}\left(\eta(T-s)^{q}\right) f(s) d s+ \\
& T^{3}\left(E_{q, 2}^{3}\left(\eta T^{q}\right)-E_{q, 4}\left(\eta T^{q}\right) E_{q, 2}\left(\eta T^{q}\right) E_{q, 0}\left(\eta T^{q}\right)\right) \int_{0}^{T}(T-s)^{q-4} E_{q, q-3}\left(\eta(T-s)^{q}\right) f(s) d s+ \\
& T^{3}\left(E_{q, 3}^{2}\left(\eta T^{q}\right) E_{q, 0}\left(\eta T^{q}\right)+E_{q, 4}\left(\eta T^{q}\right)\left(1-E_{q}\left(\eta T^{q}\right)\right)^{2}\right) \int_{0}^{T}(T-s)^{q-4} E_{q, q-3}\left(\eta(T-s)^{q}\right) f(s) d s+ \\
& 2 T^{3} E_{q, 3}\left(\eta T^{q}\right) E_{q, 2}\left(\eta T^{q}\right)\left(1-E_{q}\left(\eta T^{q}\right)\right) \int_{0}^{T}(T-s)^{q-4} E_{q, q-3}\left(\eta(T-s)^{q}\right) f(s) d s= \\
& \alpha_{1} \int_{0}^{T}(T-s)^{q-1} E_{q, q}\left(\eta(T-s)^{q}\right) f(s) d s+T \alpha_{2} \int_{0}^{T}(T-s)^{q-2} E_{q, q-1}\left(\eta(T-s)^{q}\right) f(s) d s+ \\
& T^{2} \alpha_{3} \int_{0}^{T}(T-s)^{q-3} E_{q, q-2}\left(\eta(T-s)^{q}\right) f(s) d s+T^{3} \alpha_{4} \int_{0}^{T}(T-s)^{q-4} E_{q, q-3}\left(\eta(T-s)^{q}\right) f(s) d s, \\
& \Delta_{2}=T^{-1}\left(E_{q, 0}\left(\eta T^{q}\right)\left(1-E_{q}\left(\eta T^{q}\right)\right)^{2}+E_{q, 2}^{2}\left(\eta T^{q}\right) E_{q,-2}\left(\eta T^{q}\right)\right) \int_{0}^{T}(T-s)^{q-1} E_{q, q}\left(\eta(T-s)^{q}\right) f(s) d s+ \\
& T^{-1}\left(E_{q, 3}\left(\eta T^{q}\right) E_{q,-1}\left(\eta T^{q}\right) E_{q, 0}\left(\eta T^{q}\right)+E_{q, 3}\left(\eta T^{q}\right) E_{q,-2}\left(\eta T^{q}\right)\left(1-E_{q}\left(\eta T^{q}\right)\right)\right) \int_{0}^{T}(T-s)^{q-1} E_{q, q}\left(\eta(T-s)^{q}\right) f(s) d s+ \\
& T^{-1}\left(-E_{q, 0}^{2}\left(\eta T^{q}\right) E_{q, 2}\left(\eta T^{q}\right)+E_{q, 2}\left(\eta T^{q}\right) E_{q,-1}\left(\eta T^{q}\right)\left(1-E_{q}\left(\eta T^{q}\right)\right)\right) \int_{0}^{T}(T-s)^{q-1} E_{q, q}\left(\eta(T-s)^{q}\right) f(s) d s+ \\
& \left(\left(1-E_{q}\left(\eta T^{q}\right)\right)^{3}-E_{q, 2}\left(\eta T^{q}\right) E_{q, 0}\left(\eta T^{q}\right)\left(1-E_{q}\left(\eta T^{q}\right)\right)\right) \int_{0}^{T}(T-s)^{q-2} E_{q, q-1}\left(\eta(T-s)^{q}\right) f(s) d s- \\
& \left(E_{q, 3}\left(\eta T^{q}\right) E_{q, 2}\left(\eta T^{q}\right) E_{q,-2}\left(\eta T^{q}\right)-E_{q, 3}\left(\eta T^{q}\right) E_{q,-1}\left(\eta T^{q}\right)\left(1-E_{q}\left(\eta T^{q}\right)\right)\right) \int_{0}^{T}(T-s)^{q-2} E_{q, q-1}\left(\eta(T-s)^{q}\right) f(s) d s+ \\
& \left(E_{q, 4}\left(\eta T^{q}\right) E_{q,-2}\left(\eta T^{q}\right)\left(1-E_{q}\left(\eta T^{q}\right)\right)-E_{q, 4}\left(\eta T^{q}\right) E_{q,-1}\left(\eta T^{q}\right) E_{q, 0}\left(\eta T^{q}\right)\right) \int_{0}^{T}(T-s)^{q-2} E_{q, q-1}\left(\eta(T-s)^{q}\right) f(s) d s+
\end{aligned}
$$




$$
\begin{aligned}
& T E_{q, 2}\left(\eta T^{q}\right)\left(1-E_{q}\left(\eta T^{q}\right)\right)^{2} \int_{0}^{T}(T-s)^{q-3} E_{q, q-2}\left(\eta(T-s)^{q}\right) f(s) d s+ \\
& T\left(2 E_{q, 3}\left(\eta T^{q}\right) E_{q, 0}\left(\eta T^{q}\right)\left(1-E_{q}\left(\eta T^{q}\right)\right)+E_{q, 3}^{2}\left(\eta T^{q}\right) E_{q,-2}\left(\eta T^{q}\right)\right) \int_{0}^{T}(T-s)^{q-3} E_{q, q-2}\left(\eta(T-s)^{q}\right) f(s) d s+ \\
& T\left(E_{q, 4}\left(\eta T^{q}\right) E_{q, 0}^{2}\left(\eta T^{q}\right)-E_{q, 4}\left(\eta T^{q}\right) E_{q, 2}\left(\eta T^{q}\right) E_{q,-2}\left(\eta T^{q}\right)\right) \int_{0}^{T}(T-s)^{q-3} E_{q, q-2}\left(\eta(T-s)^{q}\right) f(s) d s+ \\
& T^{2}\left(E_{q, 2}^{2}\left(\eta T^{q}\right)\left(1-E_{q}\left(\eta T^{q}\right)\right)+E_{q, 3}\left(\eta T^{q}\right)\left(1-E_{q}\left(\eta T^{q}\right)\right)^{2}\right) \int_{0}^{T}(T-s)^{q-4} E_{q, q-3}\left(\eta(T-s)^{q}\right) f(s) d s+ \\
& T^{2}\left(-E_{q, 3}^{2}\left(\eta T^{q}\right) E_{q,-1}\left(\eta T^{q}\right)+E_{q, 3}\left(\eta T^{q}\right) E_{q, 0}\left(\eta T^{q}\right) E_{q, 2}\left(\eta T^{q}\right)\right) \int_{0}^{T}(T-s)^{q-4} E_{q, q-3}\left(\eta(T-s)^{q}\right) f(s) d s+ \\
& T^{2}\left(E_{q, 4}\left(\eta T^{q}\right) E_{q, 2}\left(\eta T^{q}\right) E_{q,-1}\left(\eta T^{q}\right)+E_{q, 4}\left(\eta T^{q}\right) E_{q, 0}\left(\eta T^{q}\right)\left(1-E_{q}\left(\eta T^{q}\right)\right)\right) \int_{0}^{T}(T-s)^{q-4} E_{q, q-3}\left(\eta(T-s)^{q}\right) f(s) d s= \\
& T^{-1} \alpha_{5} \int_{0}^{T}(T-s)^{q-1} E_{q, q}\left(\eta(T-s)^{q}\right) f(s) d s+\alpha_{6} \int_{0}^{T}(T-s)^{q-2} E_{q, q-1}\left(\eta(T-s)^{q}\right) f(s) d s+ \\
& T \alpha_{7} \int_{0}^{T}(T-s)^{q-3} E_{q, q-2}\left(\eta(T-s)^{q}\right) f(s) d s+T^{2} \alpha_{3} \int_{0}^{T}(T-s)^{q-4} E_{q, q-3}\left(\eta(T-s)^{q}\right) f(s) d s, \\
& \Delta_{3}=T^{-2}\left(E_{q, 0}^{2}\left(\eta T^{q}\right)\left(1-E_{q}\left(\eta T^{q}\right)\right)-E_{q, 3}\left(\eta T^{q}\right) E_{q,-1}^{2}\left(\eta T^{q}\right)\right) \int_{0}^{T}(T-s)^{q-1} E_{q, q}\left(\eta(T-s)^{q}\right) f(s) d s+ \\
& T^{-2}\left(E_{q, 2}\left(\eta T^{q}\right) E_{q,-2}\left(\eta T^{q}\right)\left(1-E_{q}\left(\eta T^{q}\right)\right)+E_{q, 3}\left(\eta T^{q}\right) E_{q, 0}\left(\eta T^{q}\right) E_{q,-2}\left(\eta T^{q}\right)\right) \int_{0}^{T}(T-s)^{q-1} E_{q, q}\left(\eta(T-s)^{q}\right) f(s) d s+ \\
& T^{-2}\left(E_{q, 0}\left(\eta T^{q}\right) E_{q, 2}\left(\eta T^{q}\right) E_{q,-1}\left(\eta T^{q}\right)+E_{q,-1}\left(\eta T^{q}\right)\left(1-E_{q}\left(\eta T^{q}\right)\right)^{2}\right) \int_{0}^{T}(T-s)^{q-1} E_{q, q}\left(\eta(T-s)^{q}\right) f(s) d s+ \\
& T^{-1} 2 E_{q, 2}\left(\eta T^{q}\right) E_{q,-1}\left(\eta T^{q}\right)\left(1-E_{q}\left(\eta T^{q}\right)\right) \int_{0}^{T}(T-s)^{q-2} E_{q, q-1}\left(\eta(T-s)^{q}\right) f(s) d s+ \\
& T^{-1}\left(E_{q, 0}\left(\eta T^{q}\right)\left(1-E_{q}\left(\eta T^{q}\right)\right)^{2}+E_{q, 2}^{2}\left(\eta T^{q}\right) E_{q,-2}\left(\eta T^{q}\right)\right) \int_{0}^{T}(T-s)^{q-2} E_{q, q-1}\left(\eta(T-s)^{q}\right) f(s) d s+ \\
& T^{-1}\left(E_{q, 4}\left(\eta T^{q}\right) E_{q,-1}^{2}\left(\eta T^{q}\right)-E_{q, 4}\left(\eta T^{q}\right) E_{q, 0}\left(\eta T^{q}\right) E_{q,-2}\left(\eta T^{q}\right)\right) \int_{0}^{T}(T-s)^{q-2} E_{q, q-1}\left(\eta(T-s)^{q}\right) f(s) d s+ \\
& \left(\left(1-E_{q}\left(\eta T^{q}\right)\right)^{3}-E_{q, 3}\left(\eta T^{q}\right) E_{q,-1}\left(\eta T^{q}\right)\left(1-E_{q}\left(\eta T^{q}\right)\right)\right) \int_{0}^{T}(T-s)^{q-3} E_{q, q-2}\left(\eta(T-s)^{q}\right) f(s) d s- \\
& \left(E_{q, 2}\left(\eta T^{q}\right) E_{q, 0}\left(\eta T^{q}\right)\left(1-E_{q}\left(\eta T^{q}\right)\right)+E_{q, 2}\left(\eta T^{q}\right) E_{q, 3}\left(\eta T^{q}\right) E_{q,-2}\left(\eta T^{q}\right)\right) \int_{0}^{T}(T-s)^{q-3} E_{q, q-2}\left(\eta(T-s)^{q}\right) f(s) d s- \\
& \left(E_{q, 4}\left(\eta T^{q}\right) E_{q,-2}\left(\eta T^{q}\right)\left(1-E_{q}\left(\eta T^{q}\right)\right)+E_{q, 4}\left(\eta T^{q}\right) E_{q, 0}\left(\eta T^{q}\right) E_{q,-1}\left(\eta T^{q}\right)\right) \int_{0}^{T}(T-s)^{q-3} E_{q, q-2}\left(\eta(T-s)^{q}\right) f(s) d s+ \\
& T\left(E_{q, 3}\left(\eta T^{q}\right) E_{q, 0}\left(\eta T^{q}\right)\left(1-E_{q}\left(\eta T^{q}\right)\right)+E_{q, 2}\left(\eta T^{q}\right)\left(1-E_{q}\left(\eta T^{q}\right)\right)^{2}\right) \int_{0}^{T}(T-s)^{q-4} E_{q, q-3}\left(\eta(T-s)^{q}\right) f(s)+ \\
& T\left(E_{q, 2}\left(\eta T^{q}\right) E_{q, 3}\left(\eta T^{q}\right) E_{q,-1}\left(\eta T^{q}\right)-E_{q, 2}^{2}\left(\eta T^{q}\right) E_{q, 0}\left(\eta T^{q}\right)\right) \int_{0}^{T}(T-s)^{q-4} E_{q, q-3}\left(\eta(T-s)^{q}\right) f(s)+ \\
& T\left(E_{q, 4}\left(\eta T^{q}\right) E_{q, 0}^{2}\left(\eta T^{q}\right)+E_{q, 4}\left(\eta T^{q}\right) E_{q,-1}\left(\eta T^{q}\right)\left(1-E_{q}\left(\eta T^{q}\right)\right)\right) \int_{0}^{T}(T-s)^{q-4} E_{q, q-3}\left(\eta(T-s)^{q}\right) f(s)= \\
& T^{-2} \alpha_{8} \int_{0}^{T}(T-s)^{q-1} E_{q, q}\left(\eta(T-s)^{q}\right) f(s) d s+T^{-1} \alpha_{9} \int_{0}^{T}(T-s)^{q-2} E_{q, q-1}\left(\eta(T-s)^{q}\right) f(s) d s+ \\
& \alpha_{6} \int_{0}^{T}(T-s)^{q-3} E_{q, q-2}\left(\eta(T-s)^{q}\right) f(s) d s+T \alpha_{2} \int_{0}^{T}(T-s)^{q-4} E_{q, q-3}\left(\eta(T-s)^{q}\right) f(s), \\
& \Delta_{4}=T^{-3}\left(E_{q, 0}^{3}\left(\eta T^{q}\right)+E_{q,-2}\left(\eta T^{q}\right)\left(1-E_{q}\left(\eta T^{q}\right)\right)^{2}\right) \int_{0}^{T}(T-s)^{q-1} E_{q, q}\left(\eta(T-s)^{q}\right) f(s) d s+
\end{aligned}
$$




$$
\begin{aligned}
& T^{-3}\left(E_{q, 2}\left(\eta T^{q}\right) E_{q,-1}^{2}\left(\eta T^{q}\right)-E_{q, 2}\left(\eta T^{q}\right) E_{q, 0}\left(\eta T^{q}\right) E_{q,-2}\left(\eta T^{q}\right)\right) \int_{0}^{T}(T-s)^{q-1} E_{q, q}\left(\eta(T-s)^{q}\right) f(s) d s+ \\
& T^{-3}\left(E_{q, 0}\left(\eta T^{q}\right) E_{q,-1}\left(\eta T^{q}\right)\left(1-E_{q}\left(\eta T^{q}\right)\right)+E_{q,-1}\left(\eta T^{q}\right) E_{q, 0}\left(\eta T^{q}\right)\left(1-E_{q}\left(\eta T^{q}\right)\right)\right) \int_{0}^{T}(T-s)^{q-1} E_{q, q}\left(\eta(T-s)^{q}\right) f(s) d s+ \\
& T^{-2}\left(E_{q, 0}^{2}\left(\eta T^{q}\right)\left(1-E_{q}\left(\eta T^{q}\right)\right)+E_{q,-1}\left(\eta T^{q}\right)\left(1-E_{q}\left(\eta T^{q}\right)\right)^{2}\right) \int_{0}^{T}(T-s)^{q-2} E_{q, q-1}\left(\eta(T-s)^{q}\right) f(s) d s+ \\
& T^{-2}\left(E_{q, 2}\left(\eta T^{q}\right) E_{q,-1}\left(\eta T^{q}\right) E_{q, 0}\left(\eta T^{q}\right)+E_{q, 2}\left(\eta T^{q}\right) E_{q,-2}\left(\eta T^{q}\right)\left(1-E_{q}\left(\eta T^{q}\right)\right)\right) \int_{0}^{T}(T-s)^{q-2} E_{q, q-1}\left(\eta(T-s)^{q}\right) f(s) d s+ \\
& T^{-2}\left(-E_{q, 3}\left(\eta T^{q}\right) E_{q,-1}^{2}\left(\eta T^{q}\right)+E_{q, 3}\left(\eta T^{q}\right) E_{q, 0}\left(\eta T^{q}\right) E_{q,-2}\left(\eta T^{q}\right)\right) \int_{0}^{T}(T-s)^{q-2} E_{q, q-1}\left(\eta(T-s)^{q}\right) f(s) d s+ \\
& T^{-1}\left(E_{q, 2}\left(\eta T^{q}\right) E_{q,-1}\left(\eta T^{q}\right)\left(1-E_{q}\left(\eta T^{q}\right)\right)+E_{q, 0}\left(\eta T^{q}\right)\left(1-E_{q}\left(\eta T^{q}\right)\right)^{2}\right) \int_{0}^{T}(T-s)^{q-3} E_{q, q-2}\left(\eta(T-s)^{q}\right) f(s) d s+ \\
& T^{-1}\left(E_{q, 2}^{2}\left(\eta T^{q}\right) E_{q,-2}\left(\eta T^{q}\right)-E_{q, 2}\left(\eta T^{q}\right) E_{q, 0}^{2}\left(\eta T^{q}\right)\right) \int_{0}^{T}(T-s)^{q-3} E_{q, q-2}\left(\eta(T-s)^{q}\right) f(s) d s+ \\
& T^{-1}\left(E_{q, 3}\left(\eta T^{q}\right) E_{q,-2}\left(\eta T^{q}\right)\left(1-E_{q}\left(\eta T^{q}\right)\right)+E_{q, 3}\left(\eta T^{q}\right) E_{q, 0}\left(\eta T^{q}\right) E_{q,-1}\left(\eta T^{q}\right)\right) \int_{0}^{T}(T-s)^{q-3} E_{q, q-2}\left(\eta(T-s)^{q}\right) f(s) d s+ \\
& \left(\left(1-E_{q}\left(\eta T^{q}\right)\right)^{3}-E_{q, 2}\left(\eta T^{q}\right) E_{q, 0}\left(\eta T^{q}\right)\left(1-E_{q}\left(\eta T^{q}\right)\right)\right) \int_{0}^{T}(T-s)^{q-4} E_{q, q-3}\left(\eta(T-s)^{q}\right) f(s) d s- \\
& \left(E_{q, 2}\left(\eta T^{q}\right) E_{q, 0}\left(\eta T^{q}\right)\left(1-E_{q}\left(\eta T^{q}\right)\right)+E_{q, 2}^{2}\left(\eta T^{q}\right) E_{q,-1}\left(\eta T^{q}\right)\right) \int_{0}^{T}(T-s)^{q-4} E_{q, q-3}\left(\eta(T-s)^{q}\right) f(s) d s- \\
& \left(E_{q, 3}\left(\eta T^{q}\right) E_{q, 0}^{2}\left(\eta T^{q}\right) E_{q, 3}\left(\eta T^{q}\right) E_{q,-1}\left(\eta T^{q}\right)\left(1-E_{q}\left(\eta T^{q}\right)\right)\right) \int_{0}^{T}(T-s)^{q-4} E_{q, q-3}\left(\eta(T-s)^{q}\right) f(s) d s= \\
& T^{-3} \alpha_{10} \int_{0}^{T}(T-s)^{q-1} E_{q, q}\left(\eta(T-s)^{q}\right) f(s) d s+T^{-2} \alpha_{8} \int_{0}^{T}(T-s)^{q-2} E_{q, q-1}\left(\eta(T-s)^{q}\right) f(s) d s+ \\
& T^{-1} \alpha_{5} \int_{0}^{T}(T-s)^{q-3} E_{q, q-2}\left(\eta(T-s)^{q}\right) f(s) d s+\alpha_{1} \int_{0}^{T}(T-s)^{q-4} E_{q, q-3}\left(\eta(T-s)^{q}\right) f(s) d s .
\end{aligned}
$$

Таким образом, получаем следующие значения:

$$
\begin{gathered}
c_{1}=\frac{\Delta_{1}}{\Delta}=\alpha^{-1} \alpha_{1} \int_{0}^{T}(T-s)^{q-1} E_{q, q}\left(\eta(T-s)^{q}\right) f(s) d s+T \alpha^{-1} \alpha_{2} \int_{0}^{T}(T-s)^{q-2} E_{q, q-1}\left(\eta(T-s)^{q}\right) f(s) d s+ \\
T^{2} \alpha^{-1} \alpha_{3} \int_{0}^{T}(T-s)^{q-3} E_{q, q-2}\left(\eta(T-s)^{q}\right) f(s) d s+T^{3} \alpha^{-1} \alpha_{4} \int_{0}^{T}(T-s)^{q-4} E_{q, q-3}\left(\eta(T-s)^{q}\right) f(s) d s, \\
c_{2}=\frac{\Delta_{2}}{\Delta}=T^{-1} \alpha^{-1} \alpha_{5} \int_{0}^{T}(T-s)^{q-1} E_{q, q}\left(\eta(T-s)^{q}\right) f(s) d s+\alpha^{-1} \alpha_{6} \int_{0}^{T}(T-s)^{q-2} E_{q, q-1}\left(\eta(T-s)^{q}\right) f(s) d s+ \\
T^{-1} \alpha_{7} \int_{0}^{T}(T-s)^{q-3} E_{q, q-2}\left(\eta(T-s)^{q}\right) f(s) d s+T^{2} \alpha^{-1} \alpha_{3} \int_{0}^{T}(T-s)^{q-4} E_{q, q-3}\left(\eta(T-s)^{q}\right) f(s) d s, \\
c_{3}=\frac{\Delta_{3}}{\Delta}=T^{-2} \alpha^{-1} \alpha_{8} \int_{0}^{T}(T-s)^{q-1} E_{q, q}\left(\eta(T-s)^{q}\right) f(s) d s+T^{-1} \alpha^{-1} \alpha_{9} \int_{0}^{T}(T-s)^{q-2} E_{q, q-1}\left(\eta(T-s)^{q}\right) f(s) d s+ \\
\alpha^{-1} \alpha_{6} \int_{0}^{T}(T-s)^{q-3} E_{q, q-2}\left(\eta(T-s)^{q}\right) f(s) d s+T \alpha^{-1} \alpha_{2} \int_{0}^{T}(T-s)^{q-4} E_{q, q-3}\left(\eta(T-s)^{q}\right) f(s), \\
c_{4}=\frac{\Delta_{4}}{\Delta}=T^{-3} \alpha^{-1} \alpha_{10} \int_{0}^{T}(T-s)^{q-1} E_{q, q}\left(\eta(T-s)^{q}\right) f(s) d s+T^{-2} \alpha^{-1} \alpha_{8} \int_{0}^{T}(T-s)^{q-2} E_{q, q-1}\left(\eta(T-s)^{q}\right) f(s) d s+ \\
T^{-1} \alpha^{-1} \alpha_{5} \int_{0}^{T}(T-s)^{q-3} E_{q, q-2}\left(\eta(T-s)^{q}\right) f(s) d s+\alpha^{-1} \alpha_{1} \int_{0}^{T}(T-s)^{q-4} E_{q, q-3}\left(\eta(T-s)^{q}\right) f(s) d s .
\end{gathered}
$$


Подставляя найденные коэффициенты в формулу для решения, мы получаем

$$
\begin{gathered}
x(t)= \\
\alpha^{-1}\left(E_{q}\left(\eta t^{q}\right) \alpha_{1}+t E_{q, 2}\left(\eta t^{q}\right) T^{-1} \alpha_{5}+t^{2} E_{q, 3}\left(\eta t^{q}\right) T^{-2} \alpha_{8}+t^{3} E_{q, 4}\left(\eta t^{q}\right) T^{-3} \alpha_{10}\right) \int_{0}^{T}(T-s)^{q-1} E_{q, q}\left(\eta(T-s)^{q}\right) f(s) d s+ \\
\alpha^{-1}\left(E_{q}\left(\eta t^{q}\right) T \alpha_{2}+t E_{q, 2}\left(\eta t^{q}\right) \alpha_{6}+t^{2} E_{q, 3}\left(\eta t^{q}\right) T^{-1} \alpha_{9}+t^{3} E_{q, 4}\left(\eta t^{q}\right) T^{-2} \alpha_{8}\right) \int_{0}^{T}(T-s)^{q-2} E_{q, q-1}\left(\eta(T-s)^{q}\right) f(s) d s+ \\
\alpha^{-1}\left(E_{q}\left(\eta t^{q}\right) T^{2} \alpha_{3}+t E_{q, 2}\left(\eta t^{q}\right) T \alpha_{7}+t^{2} E_{q, 3}\left(\eta t^{q}\right) \alpha_{6}+t^{3} E_{q, 4}\left(\eta t^{q}\right) T^{-1} \alpha_{5}\right) \int_{0}^{T}(T-s)^{q-3} E_{q, q-2}\left(\eta(T-s)^{q}\right) f(s) d s+ \\
\alpha^{-1}\left(E_{q}\left(\eta t^{q}\right) T^{3} \alpha_{4}+t E_{q, 2}\left(\eta t^{q}\right) T^{2} \alpha_{3}+t^{2} E_{q, 3}\left(\eta t^{q}\right) T \alpha_{2}+t^{3} E_{q, 4}\left(\eta t^{q}\right) \alpha_{1}\right) \int_{0}^{T}(T-s)^{q-4} E_{q, q-3}\left(\eta(T-s)^{q}\right) f(s) d s+ \\
\int_{0}^{t}(t-s)^{q-1} E_{q, q}\left(\eta(t-s)^{q}\right) f(s) d s=\int_{0}^{T} G(t, s) f(s) d s .
\end{gathered}
$$

Для того, чтобы установить аналогичный результат для функции $f \in L^{\infty}([0, T] ; E)$, нам потребуются следующие утверждения.

Лемма 3.2. Для всякой функции $f \in L^{\infty}([0, T] ; E)$ существует последовательность $\left\{f_{n}\right\} \subset C([0, T] ; E)$ такая, что $f_{n}(t) \rightarrow f(t)$ во всех точках Лебега функции $f$ из $[0, T]$, причем $\left\|f_{n}\right\|_{C([0, T] ; E)} \leq\|f\|_{L^{\infty}([0, T] ; E)}$.

Примером такой последовательности может послужить следующая, построенная на основе проектора Стеклова

$$
\begin{gathered}
f_{n}(t)=\left\{\begin{array}{l}
\frac{1}{2 n} \int_{t-\frac{1}{n}}^{t+\frac{1}{n}} \hat{f}(s) d s, t \in[0, T] ; \\
0, t \notin[0, T],
\end{array}\right. \\
\hat{f}(t)=\left\{\begin{array}{l}
f(t), t \in[0, T] ; \\
0, t \notin[0, T] .
\end{array}\right.
\end{gathered}
$$

Лемма 3.3. (см. [8]) Для всякой функции $f \in L^{\infty}([0, T] ; E)$ множество ее точек Лебега есть множество полной меры для $[0, T]$.

Таким образом, мы можем построить функцию Грина для краевой задачи (4), (2), имеющую тот же вид, что и в лемме 3.1 , но уже при условии, что $f \in L^{\infty}([0, T] ; E)$.

Будем полагать, что мультиотображение $F:[0, T] \times E \rightarrow K v(E)$ из задачи (1)-(2) удовлетворяет следующим условиям:

$(F 1)$ для всех $x \in E$ мультифункция $F(\cdot, x):[0, T] \rightarrow K v(E)$ допускает измеримое сечение;

$(F 2)$ для п.в. $t \in[0, T]$ многозначное отображение $F(t, \cdot): E \rightarrow K v(E)$ - полунепрерывно сверху;

(F3) для каждого $r>0$ существует функция $\omega_{r} \in L_{+}^{\infty}([0, T])$ такая, что для любого $x \in E$ с $\|x\|_{E}<r$, мы имеем

$$
\|F(t, x)\|_{E} \leq \omega_{r}(t) ;
$$

(F4) существует функция $\mu \in L_{+}^{\infty}([0, T])$ такая, что для любого ограниченного множества $\Omega \subset E$, мы имеем

$$
\chi(F(t, \Omega)) \leq \mu(t) \chi(\Omega),
$$

для п.в. $t \in[0, T]$, где $\chi-$ МНК Хаусдорфа в $E$.

Для $x \in C([0, T] ; E)$ введем в рассмотрение мультифункцию:

$$
\Phi_{F}:[0, T] \rightarrow K v(E), \quad \Phi_{F}(t)=F(t, x(t)) .
$$

Из условий $(F 1)-(F 3)$ следует (см., [15], теорема 1.3.5), что мультифункция $\Phi_{F}$ является $L^{p}$-интегрируемой для любого $p \geq 1$.

Для решения нашей задачи мы будем использовать суперпозиционный мультиоператор $\mathcal{P}_{F}^{\infty}$ : $C([0, T] ; E) \multimap L^{\infty}([0, T] ; E)$, определенный следующим образом:

$$
\mathcal{P}_{F}^{\infty}(x)=\mathcal{S}_{\Phi_{F}}^{\infty} .
$$

Рассмотрим мультиоператор Г, заданный следующим образом:

$$
\Gamma x(t)=\int_{0}^{T} G(t, s) f(s) d s, \quad \text { где } \quad f \in \mathcal{P}_{F}^{\infty}(x) .
$$

Из условий $(F 1)-(F 4)$ следует, что для функции $x \in C([0, T] ; E)$, функция $f \in L^{\infty}([0, T] ; E)$. При этом из определения функции Грина следует, что для любого $t \in[0, T]$ и $3<q<4: G(\cdot, s) \in L^{p}([0, T]), p \geq 1$, и функция Грина теряет непрерывность только в точке $s=T$, поэтому $\Gamma: C([0, T] ; E) \multimap C([0, T] ; E)$. 
Если функция $x \in C([0, T] ; E)$ является решением задачи (1)-(2), то она является неподвижной точкой мультиоператора Г. Поэтому мы в дальнейшем будем доказывать существование неподвижных точек мультиоператора Г.

Покажем, что если выполняется условие $(A)$, функция Грина $G$ не меняет знак на квадрате $[0, T] \times$ $[0, T]$; тогда справедливо равенство

$$
\int_{0}^{T}|G(t, s)| d s=\frac{1}{\eta}
$$

Действительно,

$$
\begin{gathered}
\int_{0}^{T} G(t, s) d s= \\
\alpha^{-1}\left(E_{q}\left(\eta t^{q}\right) \alpha_{1}+t E_{q, 2}\left(\eta t^{q}\right) T^{-1} \alpha_{5}+t^{2} E_{q, 3}\left(\eta t^{q}\right) T^{-2} \alpha_{8}+t^{3} E_{q, 4}\left(\eta t^{q}\right) T^{-3} \alpha_{10}\right) \int_{0}^{T}(T-s)^{q-1} E_{q, q}\left(\eta(T-s)^{q}\right) d s+ \\
\alpha^{-1}\left(E_{q}\left(\eta t^{q}\right) T \alpha_{2}+t E_{q, 2}\left(\eta t^{q}\right) \alpha_{6}+t^{2} E_{q, 3}\left(\eta t^{q}\right) T^{-1} \alpha_{9}+t^{3} E_{q, 4}\left(\eta t^{q}\right) T^{-2} \alpha_{8}\right) \int_{0}^{T}(T-s)^{q-2} E_{q, q-1}\left(\eta(T-s)^{q}\right) d s+ \\
\alpha^{-1}\left(E_{q}\left(\eta t^{q}\right) T^{2} \alpha_{3}+t E_{q, 2}\left(\eta t^{q}\right) T \alpha_{7}+t^{2} E_{q, 3}\left(\eta t^{q}\right) \alpha_{6}+t^{3} E_{q, 4}\left(\eta t^{q}\right) T^{-1} \alpha_{5}\right) \int_{0}^{T}(T-s)^{q-3} E_{q, q-2}\left(\eta(T-s)^{q}\right) d s+ \\
\alpha^{-1}\left(E_{q}\left(\eta t^{q}\right) T^{3} \alpha_{4}+t E_{q, 2}\left(\eta t^{q}\right) T^{2} \alpha_{3}+t^{2} E_{q, 3}\left(\eta t^{q}\right) T \alpha_{2}+t^{3} E_{q, 4}\left(\eta t^{q}\right) \alpha_{1}\right) \int_{0}^{T}(T-s)^{q-4} E_{q, q-3}\left(\eta(T-s)^{q}\right) d s+ \\
\int_{0}^{t}(t-s)^{q-1} E_{q, q}\left(\eta(t-s)^{q}\right) d s .
\end{gathered}
$$

Вычислим интегралы в последнем выражении на основе формулы (8):

$$
\begin{gathered}
\int_{0}^{T}(T-s)^{q-1} E_{q, q}\left(\eta(T-s)^{q}\right) d s=-\int_{0}^{T}(T-s)^{q-1} E_{q, q}\left(\eta(T-s)^{q}\right) d(T-s)=\int_{0}^{T} y^{q-1} E_{q, q}\left(\eta y^{q}\right) d y= \\
T^{q} E_{q, q+1}\left(\eta T^{q}\right) .
\end{gathered}
$$

Таким образом, мы получаем

$$
\begin{gathered}
\int_{0}^{T}(T-s)^{q-4} E_{q, q-3}\left(\eta(T-s)^{q}\right) d s=T^{q-3} E_{q, q-2}\left(\eta T^{q}\right), \\
\int_{0}^{T}(T-s)^{q-3} E_{q, q-2}\left(\eta(T-s)^{q}\right) d s=T^{q-2} E_{q, q-1}\left(\eta T^{q}\right), \\
\int_{0}^{T}(T-s)^{q-2} E_{q, q-1}\left(\eta(T-s)^{q}\right) d s=T^{q-1} E_{q, q}\left(\eta T^{q}\right), \\
\int_{0}^{t}(t-s)^{q-1} E_{q, q}\left(\eta(t-s)^{q}\right) d s=t^{q} E_{q, q+1}\left(\eta t^{q}\right) .
\end{gathered}
$$

Заметим, что если взять $\beta=1$ в формуле (6), то мы получим

$$
\begin{gathered}
E_{q}\left(\eta T^{q}\right)=\frac{1}{\Gamma(1)}+\eta T^{q} E_{q, q+1}\left(\eta T^{q}\right)=1+\eta T^{q} E_{q, q+1}\left(\eta T^{q}\right) \\
E_{q}\left(\eta t^{q}\right)=\frac{1}{\Gamma(1)}+\eta t^{q} E_{q, q+1}\left(\eta t^{q}\right)=1+\eta t^{q} E_{q, q+1}\left(\eta t^{q}\right) .
\end{gathered}
$$

Воспользовавшись свойством (3), считая $\beta=0, \beta=-1$ и $\beta=-2$ соответственно в (6), мы имеем

$$
\begin{gathered}
E_{q, 0}\left(\eta T^{q}\right)=\frac{1}{\Gamma(0)}+\eta T^{q} E_{q, q}\left(\eta T^{q}\right)=\eta T^{q} E_{q, q}\left(\eta T^{q}\right) \\
E_{q,-1}\left(\eta T^{q}\right)=\frac{1}{\Gamma(-1)}+\eta T^{q} E_{q, q-1}\left(\eta T^{q}\right)=\eta T^{q} E_{q, q-1}\left(\eta T^{q}\right) \\
E_{q,-2}\left(\eta T^{q}\right)=\frac{1}{\Gamma(-2)}+\eta T^{q} E_{q, q-2}\left(\eta T^{q}\right)=\eta T^{q} E_{q, q-2}\left(\eta T^{q}\right) .
\end{gathered}
$$

Таким образом, мы имеем следующие равенства

$$
\int_{0}^{T}(T-s)^{q-1} E_{q, q}\left(\eta(T-s)^{q}\right) d s=T^{q} \frac{1}{\eta T^{q}}\left(E_{q}\left(\eta T^{q}\right)-1\right)=\frac{1}{\eta}\left(E_{q}\left(\eta T^{q}\right)-1\right),
$$




$$
\begin{aligned}
& \int_{0}^{T}(T-s)^{q-2} E_{q, q-1}\left(\eta(T-s)^{q}\right) d s=\frac{1}{\eta T} E_{q, 0}\left(\eta T^{q}\right), \\
& \int_{0}^{T}(T-s)^{q-3} E_{q, q-2}\left(\eta(T-s)^{q}\right) d s=\frac{1}{\eta T^{2}} E_{q,-1}\left(\eta T^{q}\right), \\
& \int_{0}^{T}(T-s)^{q-4} E_{q, q-3}\left(\eta(T-s)^{q}\right) d s=\frac{1}{\eta T^{3}} E_{q,-2}\left(\eta T^{q}\right), \\
& \int_{0}^{t}(t-s)^{q-1} E_{q, q}\left(\eta(t-s)^{q}\right) d s=\frac{1}{\eta}\left(E_{q}\left(\eta t^{q}\right)-1\right) .
\end{aligned}
$$

Поэтому, мы получаем

$$
\begin{gathered}
\int_{0}^{T} G(t, s) d s= \\
\alpha^{-1} \eta^{-1}\left(\alpha_{1}\left(E_{q}\left(\eta T^{q}\right)-1\right)+\alpha_{2} E_{q, 0}\left(\eta T^{q}\right)+\alpha_{3} E_{q,-1}\left(\eta T^{q}\right)+\alpha_{4} E_{q,-2}\left(\eta T^{q}\right)\right) E_{q}\left(\eta t^{q}\right)+ \\
\alpha^{-1} \eta^{-1} T^{-1}\left(\alpha_{5}\left(E_{q}\left(\eta T^{q}\right)-1\right)+\alpha_{6} E_{q, 0}\left(\eta T^{q}\right)+\alpha_{7} E_{q,-1}\left(\eta T^{q}\right)+\alpha_{3} E_{q,-2}\left(\eta T^{q}\right)\right) t E_{q, 2}\left(\eta t^{q}\right)+ \\
\alpha^{-1} \eta^{-1} T^{-2}\left(\alpha_{8}\left(E_{q}\left(\eta T^{q}\right)-1\right)+\alpha_{9} E_{q, 0}\left(\eta T^{q}\right)+\alpha_{6} E_{q,-1}\left(\eta T^{q}\right)+\alpha_{2} E_{q,-2}\left(\eta T^{q}\right)\right) t^{2} E_{q, 3}\left(\eta t^{q}\right)+ \\
\alpha^{-1} \eta^{-1} T^{-3}\left(\alpha_{10}\left(E_{q}\left(\eta T^{q}\right)-1\right)+\alpha_{8} E_{q, 0}\left(\eta T^{q}\right)+\alpha_{5} E_{q,-1}\left(\eta T^{q}\right)+\alpha_{1} E_{q,-2}\left(\eta T^{q}\right)\right) t^{3} E_{q, 4}\left(\eta t^{q}\right)+ \\
\frac{1}{\eta}\left(E_{q}\left(\eta t^{q}\right)-1\right)=I_{1}+I_{2}+I_{3}+I_{4}+\frac{1}{\eta}\left(E_{q}\left(\eta t^{q}\right)-1\right) .
\end{gathered}
$$

Вычислим отдельно значение каждого слагаемого.

$$
\begin{aligned}
& I_{1}=\alpha^{-1} \eta^{-1}\left(\alpha_{1}\left(E_{q}\left(\eta T^{q}\right)-1\right)+\alpha_{2} E_{q, 0}\left(\eta T^{q}\right)+\alpha_{3} E_{q,-1}\left(\eta T^{q}\right)+\alpha_{4} E_{q,-2}\left(\eta T^{q}\right)\right) E_{q}\left(\eta t^{q}\right)= \\
& \alpha^{-1} \eta^{-1} E_{q}\left(\eta t^{q}\right)\left(-\left(1-E_{q}\left(\eta T^{q}\right)\right)^{4}+\left(1-E_{q}\left(\eta T^{q}\right)\right) E_{q, 3}\left(\eta T^{q}\right) E_{q, 0}^{2}\left(\eta T^{q}\right)\right)+ \\
& \alpha^{-1} \eta^{-1} E_{q}\left(\eta t^{q}\right)\left(\left(1-E_{q}\left(\eta T^{q}\right)\right) E_{q, 2}^{2}\left(\eta T^{q}\right) E_{q,-1}\left(\eta T^{q}\right)+E_{q, 3}\left(\eta T^{q}\right) E_{q,-1}\left(\eta T^{q}\right)\left(1-E_{q}\left(\eta T^{q}\right)\right)^{2}\right)+ \\
& \alpha^{-1} \eta^{-1} E_{q}\left(\eta t^{q}\right) 2 E_{q, 2}\left(\eta T^{q}\right) E_{q, 0}\left(\eta T^{q}\right)\left(1-E_{q}\left(\eta T^{q}\right)\right)^{2}+ \\
& \alpha^{-1} \eta^{-1} E_{q}\left(\eta t^{q}\right)\left(E_{q, 0}\left(\eta T^{q}\right) E_{q, 2}\left(\eta T^{q}\right)\left(1-E_{q}\left(\eta T^{q}\right)\right)^{2}-E_{q, 2}^{2}\left(\eta T^{q}\right) E_{q, 0}^{2}\left(\eta T^{q}\right)\right)+ \\
& \alpha^{-1} \eta^{-1} E_{q}\left(\eta t^{q}\right)\left(E_{q, 3}\left(\eta T^{q}\right) E_{q, 0}^{2}\left(\eta T^{q}\right)\left(1-E_{q}\left(\eta T^{q}\right)\right)+E_{q, 4}\left(\eta T^{q}\right) E_{q, 0}^{3}\left(\eta T^{q}\right)\right)+ \\
& \alpha^{-1} \eta^{-1} E_{q}\left(\eta t^{q}\right) E_{q, 0}\left(\eta T^{q}\right)\left(E_{q, 3}\left(\eta T^{q}\right) E_{q, 2}\left(\eta T^{q}\right) E_{q,-1}\left(\eta T^{q}\right)+E_{q, 4}\left(\eta T^{q}\right) E_{q,-1}\left(\eta T^{q}\right)\left(1-E_{q}\left(\eta T^{q}\right)\right)\right)+ \\
& \alpha^{-1} \eta^{-1} E_{q}\left(\eta t^{q}\right)\left(E_{q,-1}\left(\eta T^{q}\right) E_{q, 2}\left(\eta T^{q}\right) E_{q, 3}\left(\eta T^{q}\right) E_{q, 0}\left(\eta T^{q}\right)-E_{q, 3}^{2}\left(\eta T^{q}\right) E_{q,-1}^{2}\left(\eta T^{q}\right)\right)+ \\
& \alpha^{-1} \eta^{-1} E_{q}\left(\eta t^{q}\right)\left(E_{q,-1}\left(\eta T^{q}\right) E_{q, 3}\left(\eta T^{q}\right)\left(1-E_{q}\left(\eta T^{q}\right)\right)^{2}+E_{q, 4}\left(\eta T^{q}\right) E_{q, 2}\left(\eta T^{q}\right) E_{q,-1}^{2}\left(\eta T^{q}\right)\right)+ \\
& \alpha^{-1} \eta^{-1} E_{q}\left(\eta t^{q}\right)\left(E_{q,-1}\left(\eta T^{q}\right) E_{q, 2}^{2}\left(\eta T^{q}\right)\left(1-E_{q}\left(\eta T^{q}\right)\right)+E_{q,-1}\left(\eta T^{q}\right) E_{q, 4}\left(\eta T^{q}\right) E_{q, 0}\left(\eta T^{q}\right)\left(1-E_{q}\left(\eta T^{q}\right)\right)\right)+ \\
& \alpha^{-1} \eta^{-1} E_{q}\left(\eta t^{q}\right)\left(E_{q,-2}\left(\eta T^{q}\right) E_{q, 2}^{3}\left(\eta T^{q}\right)-E_{q,-2}\left(\eta T^{q}\right) E_{q, 4}\left(\eta T^{q}\right) E_{q, 2}\left(\eta T^{q}\right) E_{q, 0}\left(\eta T^{q}\right)\right)+ \\
& \alpha^{-1} \eta^{-1} E_{q}\left(\eta t^{q}\right)\left(E_{q,-2}\left(\eta T^{q}\right) E_{q, 3}^{2}\left(\eta T^{q}\right) E_{q, 0}\left(\eta T^{q}\right)+E_{q,-2}\left(\eta T^{q}\right) E_{q, 4}\left(\eta T^{q}\right)\left(1-E_{q}\left(\eta T^{q}\right)\right)^{2}\right)+ \\
& \alpha^{-1} \eta^{-1} E_{q}\left(\eta t^{q}\right) E_{q,-2}\left(\eta T^{q}\right) 2 E_{q, 3}\left(\eta T^{q}\right) E_{q, 2}\left(\eta T^{q}\right)\left(1-E_{q}\left(\eta T^{q}\right)\right)=-\alpha^{-1} \eta^{-1} E_{q}\left(\eta t^{q}\right) \alpha=-\eta^{-1} E_{q}\left(\eta t^{q}\right) ; \\
& I_{2}=\alpha^{-1} \eta^{-1} T^{-1}\left(\alpha_{5}\left(E_{q}\left(\eta T^{q}\right)-1\right)+\alpha_{6} E_{q, 0}\left(\eta T^{q}\right)+\alpha_{7} E_{q,-1}\left(\eta T^{q}\right)+\alpha_{3} E_{q,-2}\left(\eta T^{q}\right)\right) t E_{q, 2}\left(\eta t^{q}\right)= \\
& \alpha^{-1} \eta^{-1} T^{-1} t E_{q, 2}\left(\eta t^{q}\right)\left(-E_{q, 0}\left(\eta T^{q}\right)\left(1-E_{q}\left(\eta T^{q}\right)\right)^{3}-\left(1-E_{q}\left(\eta T^{q}\right)\right) E_{q, 2}^{2}\left(\eta T^{q}\right) E_{q,-2}\left(\eta T^{q}\right)\right)+ \\
& \alpha^{-1} \eta^{-1} T^{-1} t E_{q, 2}\left(\eta t^{q}\right) E_{q, 3}\left(\eta T^{q}\right)\left(-\left(1-E_{q}\left(\eta T^{q}\right)\right) E_{q,-1}\left(\eta T^{q}\right) E_{q, 0}\left(\eta T^{q}\right)-E_{q,-2}\left(\eta T^{q}\right)\left(1-E_{q}\left(\eta T^{q}\right)\right)^{2}\right)+ \\
& \alpha^{-1} \eta^{-1} T^{-1} t E_{q, 2}\left(\eta t^{q}\right)\left(\left(1-E_{q}\left(\eta T^{q}\right)\right) E_{q, 0}^{2}\left(\eta T^{q}\right) E_{q, 2}\left(\eta T^{q}\right)-E_{q, 2}\left(\eta T^{q}\right) E_{q,-1}\left(\eta T^{q}\right)\left(1-E_{q}\left(\eta T^{q}\right)\right)^{2}\right)+
\end{aligned}
$$




$$
\begin{aligned}
& \alpha^{-1} \eta^{-1} T^{-1} t E_{q, 2}\left(\eta t^{q}\right)\left(E_{q, 0}\left(\eta T^{q}\right)\left(1-E_{q}\left(\eta T^{q}\right)\right)^{3}-E_{q, 0}^{2}\left(\eta T^{q}\right) E_{q, 2}\left(\eta T^{q}\right)\left(1-E_{q}\left(\eta T^{q}\right)\right)\right)+ \\
& \alpha^{-1} \eta^{-1} T^{-1} t E_{q, 2}\left(\eta t^{q}\right) E_{q, 0}\left(\eta T^{q}\right) E_{q, 3}\left(\eta T^{q}\right)\left(-E_{q, 2}\left(\eta T^{q}\right) E_{q,-2}\left(\eta T^{q}\right)-E_{q,-1}\left(\eta T^{q}\right)\left(1-E_{q}\left(\eta T^{q}\right)\right)\right)+ \\
& \alpha^{-1} \eta^{-1} T^{-1} t E_{q, 2}\left(\eta t^{q}\right) E_{q, 4}\left(\eta T^{q}\right)\left(E_{q, 0}\left(\eta T^{q}\right) E_{q,-2}\left(\eta T^{q}\right)\left(1-E_{q}\left(\eta T^{q}\right)\right)-E_{q, 0}^{2}\left(\eta T^{q}\right) E_{q,-1}\left(\eta T^{q}\right)\right)+ \\
& \alpha^{-1} \eta^{-1} T^{-1} t E_{q, 2}\left(\eta t^{q}\right) E_{q,-1}\left(\eta T^{q}\right)\left(E_{q, 2}\left(\eta T^{q}\right)\left(1-E_{q}\left(\eta T^{q}\right)\right)^{2}+2 E_{q, 3}\left(\eta T^{q}\right) E_{q, 0}\left(\eta T^{q}\right)\left(1-E_{q}\left(\eta T^{q}\right)\right)\right)+ \\
& \alpha^{-1} \eta^{-1} T^{-1} t E_{q, 2}\left(\eta t^{q}\right)\left(E_{q,-1}\left(\eta T^{q}\right) E_{q, 3}^{2}\left(\eta T^{q}\right) E_{q,-2}\left(\eta T^{q}\right)+E_{q,-1}\left(\eta T^{q}\right) E_{q, 4}\left(\eta T^{q}\right) E_{q, 0}^{2}\left(\eta T^{q}\right)\right) \\
& \alpha^{-1} \eta^{-1} T^{-1} t E_{q, 2}\left(\eta t^{q}\right) E_{q, 2}\left(\eta T^{q}\right) E_{q,-2}\left(\eta T^{q}\right)\left(-E_{q,-1}\left(\eta T^{q}\right) E_{q, 4}\left(\eta T^{q}\right)+E_{q, 3}\left(\eta T^{q}\right) E_{q, 0}\left(\eta T^{q}\right)\right)+ \\
& \alpha^{-1} \eta^{-1} T^{-1} t E_{q, 2}\left(\eta t^{q}\right) E_{q,-2}\left(\eta T^{q}\right)\left(-E_{q, 3}^{2}\left(\eta T^{q}\right) E_{q,-1}\left(\eta T^{q}\right)+E_{q, 3}\left(\eta T^{q}\right)\left(1-E_{q}\left(\eta T^{q}\right)\right)^{2}\right)+ \\
& \alpha^{-1} \eta^{-1} T^{-1} t E_{q, 2}\left(\eta t^{q}\right) E_{q,-2}\left(\eta T^{q}\right)\left(E_{q, 4}\left(\eta T^{q}\right) E_{q, 2}\left(\eta T^{q}\right) E_{q,-1}\left(\eta T^{q}\right)+E_{q, 2}^{2}\left(\eta T^{q}\right)\left(1-E_{q}\left(\eta T^{q}\right)\right)\right)+ \\
& \alpha^{-1} \eta^{-1} T^{-1} t E_{q, 2}\left(\eta t^{q}\right) E_{q,-2}\left(\eta T^{q}\right) E_{q, 4}\left(\eta T^{q}\right) E_{q, 0}\left(\eta T^{q}\right)\left(1-E_{q}\left(\eta T^{q}\right)\right)=0 \\
& I_{3}=\alpha^{-1} \eta^{-1} T^{-2}\left(\alpha_{8}\left(E_{q}\left(\eta T^{q}\right)-1\right)+\alpha_{9} E_{q, 0}\left(\eta T^{q}\right)+\alpha_{6} E_{q,-1}\left(\eta T^{q}\right)+\alpha_{2} E_{q,-2}\left(\eta T^{q}\right)\right) t^{2} E_{q, 3}\left(\eta t^{q}\right)= \\
& \alpha^{-1} \eta^{-1} T^{-2} t^{2} E_{q, 3}\left(\eta t^{q}\right)\left(-E_{q, 0}^{2}\left(\eta T^{q}\right)\left(1-E_{q}\left(\eta T^{q}\right)\right)^{2}+\left(1-E_{q}\left(\eta T^{q}\right)\right) E_{q, 3}\left(\eta T^{q}\right) E_{q,-1}^{2}\left(\eta T^{q}\right)\right)+ \\
& \alpha^{-1} \eta^{-1} T^{-2} t^{2} E_{q, 3}\left(\eta t^{q}\right) E_{q,-2}\left(\eta T^{q}\right)\left(-E_{q, 2}\left(\eta T^{q}\right)\left(1-E_{q}\left(\eta T^{q}\right)\right)^{2}-\left(1-E_{q}\left(\eta T^{q}\right)\right) E_{q, 3}\left(\eta T^{q}\right) E_{q, 0}\left(\eta T^{q}\right)\right)+ \\
& \alpha^{-1} \eta^{-1} T^{-2} t^{2} E_{q, 3}\left(\eta t^{q}\right)\left(-\left(1-E_{q}\left(\eta T^{q}\right)\right) E_{q, 0}\left(\eta T^{q}\right) E_{q, 2}\left(\eta T^{q}\right) E_{q,-1}\left(\eta T^{q}\right)-E_{q,-1}\left(\eta T^{q}\right)\left(1-E_{q}\left(\eta T^{q}\right)\right)^{3}\right)+ \\
& \alpha^{-1} \eta^{-1} T^{-2} t^{2} E_{q, 3}\left(\eta t^{q}\right) E_{q, 0}\left(\eta T^{q}\right) 2 E_{q, 2}\left(\eta T^{q}\right) E_{q,-1}\left(\eta T^{q}\right)\left(1-E_{q}\left(\eta T^{q}\right)\right)+ \\
& \alpha^{-1} \eta^{-1} T^{-2} t^{2} E_{q, 3}\left(\eta t^{q}\right)\left(E_{q, 0}^{2}\left(\eta T^{q}\right)\left(1-E_{q}\left(\eta T^{q}\right)\right)^{2}+E_{q, 0}\left(\eta T^{q}\right) E_{q, 2}^{2}\left(\eta T^{q}\right) E_{q,-2}\left(\eta T^{q}\right)\right)+ \\
& \alpha^{-1} \eta^{-1} T^{-2} t^{2} E_{q, 3}\left(\eta t^{q}\right)\left(E_{q, 0}\left(\eta T^{q}\right) E_{q, 4}\left(\eta T^{q}\right) E_{q,-1}^{2}\left(\eta T^{q}\right)-E_{q, 4}\left(\eta T^{q}\right) E_{q, 0}^{2}\left(\eta T^{q}\right) E_{q,-2}\left(\eta T^{q}\right)\right)+ \\
& \alpha^{-1} \eta^{-1} T^{-2} t^{2} E_{q, 3}\left(\eta t^{q}\right)\left(E_{q,-1}\left(\eta T^{q}\right)\left(1-E_{q}\left(\eta T^{q}\right)\right)^{3}-E_{q,-1}\left(\eta T^{q}\right) E_{q, 2}\left(\eta T^{q}\right) E_{q, 0}\left(\eta T^{q}\right)\left(1-E_{q}\left(\eta T^{q}\right)\right)\right)+ \\
& \alpha^{-1} \eta^{-1} T^{-2} t^{2} E_{q, 3}\left(\eta t^{q}\right)\left(-E_{q,-1}\left(\eta T^{q}\right) E_{q, 2}\left(\eta T^{q}\right) E_{q,-2}\left(\eta T^{q}\right) E_{q, 3}\left(\eta T^{q}\right)-E_{q,-1}^{2}\left(\eta T^{q}\right)\left(1-E_{q}\left(\eta T^{q}\right)\right) E_{q, 3}\left(\eta T^{q}\right)\right)+ \\
& \alpha^{-1} \eta^{-1} T^{-2} t^{2} E_{q, 3}\left(\eta t^{q}\right) E_{q,-1}\left(\eta T^{q}\right) E_{q, 4}\left(\eta T^{q}\right) E_{q,-2}\left(\eta T^{q}\right)\left(1-E_{q}\left(\eta T^{q}\right)\right)+ \\
& \alpha^{-1} \eta^{-1} T^{-2} t^{2} E_{q, 3}\left(\eta t^{q}\right)\left(-E_{q, 4}\left(\eta T^{q}\right) E_{q,-1}^{2}\left(\eta T^{q}\right) E_{q, 0}\left(\eta T^{q}\right)+E_{q,-2}\left(\eta T^{q}\right) E_{q, 2}\left(\eta T^{q}\right)\left(1-E_{q}\left(\eta T^{q}\right)\right)^{2}\right)+ \\
& \alpha^{-1} \eta^{-1} T^{-2} t^{2} E_{q, 3}\left(\eta t^{q}\right) E_{q,-2}\left(\eta T^{q}\right)\left(-E_{q, 2}^{2}\left(\eta T^{q}\right) E_{q, 0}\left(\eta T^{q}\right)+E_{q, 3}\left(\eta T^{q}\right) E_{q, 0}\left(\eta T^{q}\right)\left(1-E_{q}\left(\eta T^{q}\right)\right)\right)+ \\
& \alpha^{-1} \eta^{-1} T^{-2} t^{2} E_{q, 3}\left(\eta t^{q}\right) E_{q,-2}\left(\eta T^{q}\right)\left(E_{q, 4}\left(\eta T^{q}\right) E_{q, 0}^{2}\left(\eta T^{q}\right)+E_{q, 3}\left(\eta T^{q}\right) E_{q, 2}\left(\eta T^{q}\right) E_{q,-1}\left(\eta T^{q}\right)\right)+ \\
& \alpha^{-1} \eta^{-1} T^{-2} t^{2} E_{q, 3}\left(\eta t^{q}\right) E_{q,-2}\left(\eta T^{q}\right) E_{q, 4}\left(\eta T^{q}\right) E_{q,-1}\left(\eta T^{q}\right)\left(1-E_{q}\left(\eta T^{q}\right)\right)=0 ; \\
& I_{4}=\alpha^{-1} \eta^{-1} T^{-3}\left(\alpha_{10}\left(E_{q}\left(\eta T^{q}\right)-1\right)+\alpha_{8} E_{q, 0}\left(\eta T^{q}\right)+\alpha_{5} E_{q,-1}\left(\eta T^{q}\right)+\alpha_{1} E_{q,-2}\left(\eta T^{q}\right)\right) t^{3} E_{q, 4}\left(\eta t^{q}\right)= \\
& \alpha^{-1} \eta^{-1} T^{-3} t^{3} E_{q, 4}\left(\eta t^{q}\right)\left(-\left(1-E_{q}\left(\eta T^{q}\right)\right) E_{q, 0}^{3}\left(\eta T^{q}\right)-E_{q,-2}\left(\eta T^{q}\right)\left(1-E_{q}\left(\eta T^{q}\right)\right)^{3}\right)+ \\
& \alpha^{-1} \eta^{-1} T^{-3} t^{3} E_{q, 4}\left(\eta t^{q}\right)\left(1-E_{q}\left(\eta T^{q}\right)\right)\left(-E_{q, 2}\left(\eta T^{q}\right) E_{q,-1}^{2}\left(\eta T^{q}\right)+E_{q, 2}\left(\eta T^{q}\right) E_{q, 0}\left(\eta T^{q}\right) E_{q,-2}\left(\eta T^{q}\right)\right)- \\
& \alpha^{-1} \eta^{-1} T^{-3} t^{3} E_{q, 4}\left(\eta t^{q}\right) E_{q, 0}\left(\eta T^{q}\right) E_{q,-1}\left(\eta T^{q}\right)\left(\left(1-E_{q}\left(\eta T^{q}\right)\right)^{2}-\left(1-E_{q}\left(\eta T^{q}\right)\right)^{2}\right)+ \\
& \alpha^{-1} \eta^{-1} T^{-3} t^{3} E_{q, 4}\left(\eta t^{q}\right)\left(E_{q, 0}^{3}\left(\eta T^{q}\right)\left(1-E_{q}\left(\eta T^{q}\right)\right)-E_{q, 0}\left(\eta T^{q}\right) E_{q, 3}\left(\eta T^{q}\right) E_{q,-1}^{2}\left(\eta T^{q}\right)\right)+ \\
& \alpha^{-1} \eta^{-1} T^{-3} t^{3} E_{q, 4}\left(\eta t^{q}\right) E_{q, 0}\left(\eta T^{q}\right) E_{q, 2}\left(\eta T^{q}\right) E_{q,-2}\left(\eta T^{q}\right)\left(1-E_{q}\left(\eta T^{q}\right)\right)+ \\
& \alpha^{-1} \eta^{-1} T^{-3} t^{3} E_{q, 4}\left(\eta t^{q}\right) E_{q, 0}^{2}\left(\eta T^{q}\right)\left(E_{q, 3}\left(\eta T^{q}\right) E_{q,-2}\left(\eta T^{q}\right)+E_{q, 2}\left(\eta T^{q}\right) E_{q,-1}\left(\eta T^{q}\right)\right)+
\end{aligned}
$$




$$
\begin{gathered}
\alpha^{-1} \eta^{-1} T^{-3} t^{3} E_{q, 4}\left(\eta t^{q}\right)\left(E_{q, 0}\left(\eta T^{q}\right) E_{q,-1}\left(\eta T^{q}\right)\left(1-E_{q}\left(\eta T^{q}\right)\right)^{2}+E_{q,-1}\left(\eta T^{q}\right) E_{q, 0}\left(\eta T^{q}\right)\left(1-E_{q}\left(\eta T^{q}\right)\right)^{2}\right)+ \\
\alpha^{-1} \eta^{-1} T^{-3} t^{3} E_{q, 4}\left(\eta t^{q}\right)\left(E_{q,-1}\left(\eta T^{q}\right) E_{q, 2}^{2}\left(\eta T^{q}\right) E_{q,-2}\left(\eta T^{q}\right)+E_{q, 3}\left(\eta T^{q}\right) E_{q,-1}^{2}\left(\eta T^{q}\right) E_{q, 0}\left(\eta T^{q}\right)\right)+ \\
\alpha^{-1} \eta^{-1} T^{-3} t^{3} E_{q, 4}\left(\eta t^{q}\right) E_{q,-1}\left(\eta T^{q}\right) E_{q, 3}\left(\eta T^{q}\right) E_{q,-2}\left(\eta T^{q}\right)\left(1-E_{q}\left(\eta T^{q}\right)\right)- \\
\alpha^{-1} \eta^{-1} T^{-3} t^{3} E_{q, 4}\left(\eta t^{q}\right)\left(E_{q,-1}\left(\eta T^{q}\right) E_{q, 0}^{2}\left(\eta T^{q}\right) E_{q, 2}\left(\eta T^{q}\right)+E_{q, 2}\left(\eta T^{q}\right) E_{q,-1}^{2}\left(\eta T^{q}\right)\left(1-E_{q}\left(\eta T^{q}\right)\right)\right)+ \\
\alpha^{-1} \eta^{-1} T^{-3} t^{3} E_{q, 4}\left(\eta t^{q}\right)\left(E_{q,-2}\left(\eta T^{q}\right)\left(1-E_{q}\left(\eta T^{q}\right)\right)^{3}-E_{q,-2}\left(\eta T^{q}\right) E_{q, 3}\left(\eta T^{q}\right) E_{q, 0}^{2}\left(\eta T^{q}\right)\right)+ \\
\alpha^{-1} \eta^{-1} T^{-3} t^{3} E_{q, 4}\left(\eta t^{q}\right) E_{q,-2}\left(\eta T^{q}\right)\left(-E_{q, 2}^{2}\left(\eta T^{q}\right) E_{q,-1}\left(\eta T^{q}\right)-E_{q, 3}\left(\eta T^{q}\right) E_{q,-1}\left(\eta T^{q}\right)\left(1-E_{q}\left(\eta T^{q}\right)\right)\right)- \\
\alpha^{-1} \eta^{-1} T^{-3} t^{3} E_{q, 4}\left(\eta t^{q}\right) E_{q,-2}\left(\eta T^{q}\right) 2 E_{q, 2}\left(\eta T^{q}\right) E_{q, 0}\left(\eta T^{q}\right)\left(1-E_{q}\left(\eta T^{q}\right)\right)=0 .
\end{gathered}
$$

Тогда

$$
\int_{0}^{T} G(t, s) d s=-\frac{1}{\eta} E_{q}\left(\eta t^{q}\right)+\frac{1}{\eta}\left(E_{q}\left(\eta t^{q}\right)-1\right)=-\frac{1}{\eta} .
$$

Для доказательства существования неподвижных точек мультиоператора Г введем в рассмотрение оператор $S: L^{\infty}([0, T] ; E) \rightarrow C([0, T] ; E)$ вида

$$
S(f)(t)=\int_{0}^{t}(t-s)^{q-1} E_{q, q}\left(\eta(t-s)^{q}\right) f(s) d s .
$$

Лемма 3.4. (см. [16]) Для каждого компактного множества $K \subset E$ и ограниченной последовательности $\left\{\xi_{n}\right\} \subset L^{\infty}([0, T] ; E)$ такой, что $\left\{\xi_{n}(t)\right\} \subset K$ для п.в. $t \in[0, T]$, слабая сходимость $\xi_{n} \rightarrow \xi_{0}$ в $L^{1}([0, T] ; E)$ влечет сходимость $S\left(\xi_{n}\right) \rightarrow S\left(\xi_{0}\right)$ в $C([0, T] ; E)$.

Лемма 3.5. (см. [16]) Пусть $\Omega \subset C([0, T] ; E)$ - непустое ограниченное множество и $\Omega(t)$ - относительно компактное подмножество $Е$ для каждого $t \in[0, T]$, тогда

$$
M=\left\{S(f)(t)=\int_{0}^{t}(t-s)^{q-1} E_{q, q}\left(\eta(t-s)^{q}\right) f(s) d s: f \in \mathcal{P}_{F}^{\infty}(x), x \in \Omega\right\}
$$

является равностепенно непрерывным множеством.

Для доказательства того, что мультиоператор Г уплотняющий, рассмотрим конус

$$
\mathbb{R}_{+}^{2}=\left\{\zeta=\left(\zeta_{1}, \zeta_{2}\right): \zeta_{1} \geq 0, \zeta_{2} \geq 0\right\}
$$

с естественным частичным порядком и введем в пространстве $C([0, T] ; E)$ следующую векторную меру некомпактности

$$
v: P b(C([0, T] ; E)) \rightarrow \mathbb{R}_{+}^{2}
$$

определенную как

$$
v(\Omega)=\left(\varphi(\Omega), \bmod _{C}(\Omega)\right),
$$

где $\varphi(\Omega)$ есть модуль послойной некомпактности

$$
\varphi(\Omega)=\sup _{t \in[0, T]} \chi(\{y(t): y \in \Omega\}),
$$

а вторая компонента - модуль равностепенной непрерывности

$$
\bmod _{C}(\Omega)=\lim _{\delta \rightarrow 0} \sup _{y \in \Omega} \max _{\left|t_{1}-t_{2}\right| \leq \delta}\left\|y\left(t_{1}\right)-y\left(t_{2}\right)\right\| .
$$

Теорема 3.1. Пусть выполняются условия $(F 1)-(F 4),(9),(A)$, предположим, что дополнительно выполняется условие

$$
\frac{\|\mu\|_{\infty}}{\eta}<1
$$

где $\mu(\cdot)$ - функция из условия (F4). Тогда мультиоператор Г является v-уплотняющим.

Доказательство. Пусть $\Omega \subset C([0, T] ; E)$ - непустое ограниченное множество такое, что

$$
v(\Gamma(\Omega)) \geq v(\Omega) .
$$

Покажем, что $\Omega$ - относительно компактно.

Из (12), следует, что

$$
\varphi(\Gamma(\Omega)) \geq \varphi(\Omega)
$$


Используя свойство $(F 4)$ и (10), мы получаем

$$
\begin{gathered}
\chi(\Gamma(\Omega)(t)) \leq \chi\left(\int_{0}^{T} G(t, s) f(s) d s: f \in \mathcal{P}_{F}^{\infty}(x), x \in \Omega\right) \leq\|\mu\|_{\infty} \int_{0}^{T}|G(t, s)| \chi(\Omega(s)) d s \leq \\
\leq\|\mu\|_{\infty} \varphi(\Omega) \int_{0}^{T}|G(t, s)| d s=\frac{\|\mu\|_{\infty}}{\eta} \varphi(\Omega) .
\end{gathered}
$$

Из последней оценки мы имеем

$$
\sup _{t \in[0, T]} \chi(\Gamma(\Omega)(t)) \leq \frac{\|\mu\|_{\infty}}{\eta} \varphi(\Omega)
$$

или что то же самое

$$
\varphi(\Gamma(\Omega)) \leq \frac{\|\mu\|_{\infty}}{\eta} \varphi(\Omega)
$$

Условия (11) и (13) вместе с последним влекут за собой равенство $\varphi(\Omega)=0$. Теперь покажем, что $\bmod _{C}(\Gamma(\Omega))=0$. Неравенство $(12)$ влечет за собой следующее $\bmod _{C}(\Gamma(\Omega)) \geq \bmod _{C}(\Omega)$. В то же время из леммы 3.5 известно, что множество функций

$$
M=\left\{S(f)(t)=\int_{0}^{t}(t-s)^{q-1} E_{q, q}\left(\eta(t-s)^{q}\right) f(s) d s: f \in \mathcal{P}_{F}^{\infty}(x), x \in \Omega\right\}
$$

является равностепенно непрерывным, поэтому $\bmod _{C}(\Gamma(\Omega))=0$, следовательно и $\bmod _{C}(\Omega)=0$, поэтому $v(\Omega)=(0,0)$, что доказывает относительную компактность множества $\Omega$.

Теорема 3.2. Мультиоператор Г является п.н.с.

Доказательство. Из аналитического задания мультиоператора Г и свойств многозначных отображений (см., например, [15]), следует, что утверждение достаточно доказать для мультиоператора $S \circ \mathcal{P}_{F}^{\infty}$.

Покажем, что мультиотображение $S \circ \mathcal{P}_{F}^{\infty}$ является квазикомпактным. Возьмем непустое компактное множество $A \subset C([0, T] ; E)$ и рассмотрим последовательность $\left\{y_{n}\right\} \subset S \circ \mathcal{P}_{F}^{\infty}(A), y_{n}=S\left(f_{n}\right)$, где $f_{n} \in \mathcal{P}_{F}^{\infty}\left(x_{n}\right)$ для произвольной последовательности $\left\{x_{n}\right\} \subset A$. Предположим, без ограничения общности, что $x_{n} \rightarrow x_{0} \in A$. Из условия (F4) следует, что последовательность $\left\{f_{n}(t)\right\} \subset E$ относительно компактна для п.в. $t \in[0, T]$, поэтому последовательность $\left\{f_{n}\right\}_{n=1}^{\infty}$ является $L^{1}$-полукомпактной. По критерию слабой относительной компактности Дистеля (см. [9]) мы можем предположить для произвольной подпоследовательности $\left\{f_{n_{k}}\right\}$, что $f_{n_{k}} \stackrel{L^{1}}{\rightarrow} f_{0}$. В силу свойств слабой замкнутости суперпозиционного мультиоператора (см. [15], лемма 5.1.1), мы получаем тогда, что $f_{0} \in \mathcal{P}_{F}^{\infty}\left(x_{0}\right)$. Теперь, применяя лемму 3.4., мы для соответствующей подпоследовательности получаем, что $y_{n_{k}} \rightarrow y_{0}=S\left(f_{0}\right) \in S \circ \mathcal{P}_{F}^{\infty}\left(x_{0}\right)$.

Аналогично рассуждая мы придем к утверждению о том, что мультиоператор $S \circ \mathcal{P}_{F}^{\infty}$ является замкнутым. Сославшись на утверждение леммы 2.2, мы получаем желаемый результат.

Теперь мы можем перейти к доказательству главного утверждения.

Теорема 3.3. При выполнении условий $(F 1),(F 2),(F 4),(9),(A)$, предположим, что условие (F3) имеет следующий вид:

$\left(F 3^{\prime}\right)$ существует функция $\omega \in L_{+}^{\infty}([0, T])$ такая, что $\|F(t, x)\|_{E} \leq \omega(t)\left(1+\|x\|_{E}\right)$.

Если $\frac{k}{\eta}<1$, где $k=\max \left\{\|\omega\|_{\infty},\|\mu\|_{\infty}\right\}$, функции $\omega$ и $\mu$ из условий $\left(F 3^{\prime}\right)$ и (F4) соответственно. Тогда задача (1)-(2) имеет решение.

Доказательство. Возьмем произвольную функцию $x \in C([0, T] ; E)$, тогда для $f \in \mathcal{P}_{F}^{\infty}(x)$ и $t \in[0, T]$ мы имеем следующую оценку:

$$
\begin{gathered}
\|\Gamma x(t)\|_{E} \leq\left\|\int_{0}^{T} G(t, s) f(s) d s\right\|_{E} \leq \int_{0}^{T}|G(t, s)|\|f(s)\|_{E} d s \leq \int_{0}^{T}|G(t, s)|\|\omega\|_{\infty}\left(1+\|x\|_{C([0, T] ; E)}\right) d s= \\
=\|\omega\|_{\infty}\left(1+\|x\|_{C([0, T] ; E)}\right) \int_{0}^{T}|G(t, s)| d s=\frac{\|\omega\|_{\infty}\left(1+\|x\|_{C([0, T] ; E)}\right)}{\eta} \leq \frac{k\left(1+\|x\|_{C([0, T] ; E)}\right)}{\eta} .
\end{gathered}
$$

Таким образом, если мы возьмем

$$
R \geq \frac{k \eta^{-1}}{1-k \eta^{-1}}
$$

тогда неравенство $\|x\|_{C([0, T] ; E)} \leq R$ влечет, что $\|\Gamma x\|_{C([0, T] ; E)} \leq R$. Таким образом, мультиоператор Г преобразует замкнутый шар $B_{R}(0) \subset C([0, T] ; E)$ в себя. Поскольку мультиоператор Г уплотняющий, по теореме 2.1 он имеет неподвижную точку, которая есть решение задачи (1) - (2). 


\section{Список литературы}

1. Афанасова М.С., Петросян Г.Г. 2019. О краевой задаче для функционально-дифференциального включения дробного порядка с общим начальным условием в банаховом пространстве. Известия вузов. Математика, 9:3-15. DOI: 10.26907/0021-3446-2019-9-3-15

2. Глушак А. В., Авад Х. К. 2013. О разрешимости абстрактного дифференциального уравнения дробного порядка с переменным оператором. СМФН. 47: 18-32.

3. Глушак А. В. 2010. Об одной обратной задаче для абстрактного дифференциального уравнения дробного порядка. Матем. заметки. 87 (5): 684-693.

4. Afanasova M., Liou Y. Ch., Obukhoskii V., Petrosyan G. 2019. On controllability for a system governed by a fractional-order semilinear functional differential inclusion in a Banach space. Journal of Nonlinear and Convex Analysis. 20: 1919-1935.

5. Bai Z., Lu H. 2005. Positive solutions for boundary-value problem of nonlinear fractional differential equation. J. Math. Anal. Appl. 311(2): 495-505. DOI:10.1016/j.jmaa.2005.02.052

6. Belmekki M., Nieto J.J., Rodriguez-Lopez R. 2009. Existence of Periodic Solution for a Nonlinear Fractional Differential Equation. Boundary Value Problems.11: 1-18. DOI:10.1155/2009/324561

7. Belmekki M., Nieto J.J., Rodriguez-Lopez R. 2014. Existence of solution to a periodic boundary value problem for a nonlinear impulsive fractional differential equation. Electronic Journal of Qualitative Theory of Differential Equations. 16: 1-27. DOI:10.14232/ejqtde.2014.1.16

8. Bogdan V.M. 2010. Generalized vectorial Lebesgue and Bochner integration theory, arXiv:1006.3881v1 [math.FA], 86.

9. Diestel J., Ruess W.M., Schachermayer W. 1993. On weak compactness in $L^{1}(\mu, X)$. Proc. Amer. Math. Soc. 118: 447-453. DOI:10.1090/S0002-9939-1993-1132408-X

10. Gorenflo R., Kilbas A.A., Mainardi F., Rogosin S.V. 2014. Mittag-Leffler Functions, Related Topics and Applications. Springer-Verlag, Berlin Heidelberg, 441.

11. Hilfer R. 2000. Applications of Fractional Calculus in Physics. World Scientific, Singapore, 90.

12. Kamenskii M., Obukhovskii V., Petrosyan G., Yao J.C. 2018. Boundary value problems for semilinear differential inclusions of fractional order in a Banach space.Applicable Analysis. 9: 571-591.

13. Kamenskii M., Obukhovskii V., Petrosyan G., Yao J.C. 2019. On a Periodic Boundary Value Problem for a Fractional Order Semilinear Functional Differential Inclusions in a Banach Space. Mathematics. 7: 5-19. DOI:10.3390/math7121146

14. Kamenskii M., Obukhovskii V., Petrosyan G., Yao J.C. 2021. On the Existence of a Unique Solution for a Class of Fractional Differential Inclusions in a Hilbert Space. Mathematics. 9(2): 136-154. DOI:10.3390/math9020136

15. Kamenskii M., Obukhovskii V., Zecca P. 2001. Condensing Multivalued Maps and Semilinear Differential Inclusions in Banach Spaces. Walter de Gruyter, Berlin-New-York, 254.

16. Kamenskii M.I., Petrosyan G.G., Wen, C.-F. 2021. An Existence Result for a Periodic Boundary Value Problem of Fractional Semilinear Differential Equations in a Banach Space. Journal of Nonlinear and Variational Analysis. 5(1): 155-177.

17. Ke T.D., Loi N.V., Obukhovskii V. 2015. Decay solutions for a class of fractional differential variational inequalities. Fract. Calc. Appl. Anal. 18(3): 531-553. DOI:10.1515/fca-2015-0033

18. Ke T.D., Obukhovskii V., Wong N.C., Yao J.C. 2013. On a class of fractional order differential inclusions with infinite delays. Applicable Anal. 92(1): 115-137. DOI:10.1080/00036811.2011.601454

19. Kilbas A.A., Srivastava H.M., Trujillo J.J. 2006. Theory and Applications of Fractional Differential Equations. Elsevier Science B.V., North-Holland Mathematics Studies, Amsterdam, 540.

20. Mainardi F., Rionero S., Ruggeri T. 1994. On the initial value problem for the fractional diffusion-wave equation, Waves and Stability in Continuous Media. World Scientific. Singapore. 246-251.

21. Obukhovskii V., Gel'man B. 2020. Multivalued Maps and Differential Inclusions. Elements of Theory and Applications. World Scientific, Hackensack, 220. 
22. Podlubny I. 1999. Fractional Differential Equations. Academic Press, San Diego, 368.

23. Shishkina E., Sitnik S. 2020. Transmutations, Singular and Fractional Differential Equations with Applications to Mathematical Physics, Mathematics in Science and Engineering. Academic Press, Elsevier, 592.

24. Shishkina E., Sitnik S., Polunin V. 2021. On sharp bounds in the T. Lyonss neo-classical inequality, which is essential for the theory of stochastic differential equations. Global and Stochastic Analysis. 8 (2): 77-86.

25. Tarasov V.E. 2010. Fractional Dynamics. Applications of Fractional Calculus to Dynamics of Particles, Fields and Media. Springer, London, New York, 522.

\section{References}

1. Afanasova M., Petrosyan G. 2019. On the boundary value problem for functional differential inclusion of fractional order with general initial condition in a Banach space. Russian Mathematics. 63: 1-12. DOI: $10.3103 /$ S1066369X19090019

2. Glushak A. V., Avad H. K. 2014. On the solvability of an abstract differential equation of fractional order with a variable operator. Journal of Mathematical Sciences. 202 (5): 637-652.

3. Glushak A. V. 2010. On an Inverse Problem for an Abstract Differential Equation of Fractional Order. Math. Notes. 87 (5): 654-662.

4. Afanasova M., Liou Y. Ch., Obukhoskii V., Petrosyan G. 2019. On controllability for a system governed by a fractional-order semilinear functional differential inclusion in a Banach space. Journal of Nonlinear and Convex Analysis. 20: 1919-1935 .

5. Bai Z., Lu H. 2005. Positive solutions for boundary-value problem of nonlinear fractional differential equation. J. Math. Anal. Appl. 311(2): 495-505. DOI:10.1016/j.jmaa.2005.02.052

6. Belmekki M., Nieto J.J., Rodriguez-Lopez R. 2009. Existence of Periodic Solution for a Nonlinear Fractional Differential Equation. Boundary Value Problems.11: 1-18. DOI:10.1155/2009/324561

7. Belmekki M., Nieto J.J., Rodriguez-Lopez R. 2014. Existence of solution to a periodic boundary value problem for a nonlinear impulsive fractional differential equation. Electronic Journal of Qualitative Theory of Differential Equations. 16: 1-27. DOI:10.14232/ejqtde.2014.1.16

8. Bogdan V.M. 2010. Generalized vectorial Lebesgue and Bochner integration theory, arXiv:1006.3881v1 [math.FA], 86.

9. Diestel J., Ruess W.M., Schachermayer W. 1993. On weak compactness in $L^{1}(\mu, X)$. Proc. Amer. Math. Soc. 118: 447-453. DOI:10.1090/S0002-9939-1993-1132408-X

10. Gorenflo R., Kilbas A.A., Mainardi F., Rogosin S.V. 2014. Mittag-Leffler Functions, Related Topics and Applications. Springer-Verlag, Berlin Heidelberg, 441.

11. Hilfer R. 2000. Applications of Fractional Calculus in Physics. World Scientific, Singapore, 90.

12. Kamenskii M., Obukhovskii V., Petrosyan G., Yao J.C. 2018. Boundary value problems for semilinear differential inclusions of fractional order in a Banach space.Applicable Analysis. 9: 571-591.

13. Kamenskii M., Obukhovskii V., Petrosyan G., Yao J.C. 2019. On a Periodic Boundary Value Problem for a Fractional Order Semilinear Functional Differential Inclusions in a Banach Space. Mathematics. 7: 5-19. DOI:10.3390/math7121146

14. Kamenskii M., Obukhovskii V., Petrosyan G., Yao J.C. 2021. On the Existence of a Unique Solution for a Class of Fractional Differential Inclusions in a Hilbert Space. Mathematics. 9(2): 136-154. DOI:10.3390/math9020136

15. Kamenskii M., Obukhovskii V., Zecca P. 2001. Condensing Multivalued Maps and Semilinear Differential Inclusions in Banach Spaces. Walter de Gruyter, Berlin-New-York, 254.

16. Kamenskii M.I., Petrosyan G.G., Wen, C.-F. 2021. An Existence Result for a Periodic Boundary Value Problem of Fractional Semilinear Differential Equations in a Banach Space. Journal of Nonlinear and Variational Analysis. 5(1): 155-177.

17. Ke T.D., Loi N.V., Obukhovskii V. 2015. Decay solutions for a class of fractional differential variational inequalities. Fract. Calc. Appl. Anal. 18(3): 531-553. DOI:10.1515/fca-2015-0033 
18. Ke T.D., Obukhovskii V., Wong N.C., Yao J.C. 2013. On a class of fractional order differential inclusions with infinite delays. Applicable Anal. 92(1): 115-137. DOI:10.1080/00036811.2011.601454

19. Kilbas A.A., Srivastava H.M., Trujillo J.J. 2006. Theory and Applications of Fractional Differential Equations. Elsevier Science B.V., North-Holland Mathematics Studies, Amsterdam, 540.

20. Mainardi F., Rionero S., Ruggeri T. 1994. On the initial value problem for the fractional diffusion-wave equation, Waves and Stability in Continuous Media. World Scientific. Singapore. 246-251.

21. Obukhovskii V., Gel'man B. 2020. Multivalued Maps and Differential Inclusions. Elements of Theory and Applications. World Scientific, Hackensack, 220.

22. Podlubny I. 1999. Fractional Differential Equations. Academic Press, San Diego, 368.

23. Shishkina E., Sitnik S. 2020. Transmutations, Singular and Fractional Differential Equations with Applications to Mathematical Physics, Mathematics in Science and Engineering. Academic Press, Elsevier, 592.

24. Shishkina E., Sitnik S., Polunin V. 2021. On sharp bounds in the T. Lyonss neo-classical inequality, which is essential for the theory of stochastic differential equations. Global and Stochastic Analysis. 8 (2): 77-86.

25. Tarasov V.E. 2010. Fractional Dynamics. Applications of Fractional Calculus to Dynamics of Particles, Fields and Media. Springer, London, New York, 522.

Конфликт интересов: о потенциальном конфликте интересов не сообщалось.

Conflict of interest: no potential conflict of interest related to this article was reported.

Получена 28.11.2021

\section{СВЕДЕНИЯ ОБ АВТОРАХ}

Петросян Гарик Гагикович - кандидат физико-математических наук, доцент, доцент кафедры высшей математики Воронежского государственного педагогического университета

(D) http://orcid.org/0000-0001-8154-6299

Улица Ленина, 86, Воронеж, 394043, Россия

E-mail: garikpetrosyan@yandex.ru

Сорока Мария Сергеевна - кандидат физико-математических наук, доцент кафедры высшей математики Воронежского государственного педагогического университета

(iD) http://orcid.org/0000-0001-8722-520X

Улица Ленина, 86, Воронеж, 394043, Россия

E-mail: marya.afanasowa@yandex.ru

\section{INFORMATION ABOUT THE AUTHORS}

Garik Petrosyan - PhD in Physics and Mathematics, Associate Professor, Associate Professor of the Department of Higher Mathematics, Voronezh State Pedagogical University, Voronezh, Russia

Maria Soroka - PhD in Physics and Mathematics, Associate Professor, Associate Professor of the Department of Higher Mathematics, Voronezh State Pedagogical University, Voronezh, Russia 